\title{
"Nano": An Emerging Avenue in Electrochemical Detection of Neurotransmitters
}

\section{Sasya Madhurantakam,*,\# Jayanth Babu Karnam,\# Dermot Brabazon, Madoka Takai, Inam UI Ahad, John Bosco Balaguru Rayappan, and Uma Maheswari Krishnan*}

School of Electrical and Electronics Engineering and Centre for Nanotechnology and Advanced Biomaterials (CeNTAB), SASTRA Deemed University, Thanjavur 613401, India

I-Form, Advanced Manufacturing Research Centre, Advanced Processing Technology Research Centre, Dublin City University, Dublin, Ireland

Department of Bioengineering, The University of Tokyo, Bunkyo-ku, Tokyo, Japan

School of Electrical and Electronics Engineering, SASTRA Deemed University, Thanjavur 613401, India

\section{Abstract}

The growing importance of nanomaterials toward the detection of neurotransmitter molecules has been chronicled in this review. Neurotransmitters (NTs) are chemicals that serve as messengers in synaptic transmission and are key players in brain functions. Abnormal levels of NTs are associated with numerous psychotic and neurodegenerative diseases. Therefore, their sensitive and robust detection is of great significance in clinical diagnostics. For more than three decades, electrochemical sensors have made a mark toward clinical detection of NTs. The superiority of these electrochemical sensors lies in their ability to enable sensitive, simple, rapid, and selective determination of analyte molecules while remaining relatively inexpensive. Additionally, these sensors are capable of being integrated in robust, portable, and miniaturized devices to establish point-of-care diagnostic platforms. Nanomaterials have emerged as promising materials with significant implications for electrochemical sensing due to their inherent capability to achieve high surface coverage, superior sensitivity, and rapid response in addition to simple device architecture and miniaturization. Considering the enormous significance of the levels of NTs in biological systems and the advances in sensing ushered in with the integration of nanotechnology in electrochemistry, the analysis of NTs by employing nanomaterials as interface materials in various matrices has emerged as an active area of research. This review explores the advancements made in the field of electrochemical sensors for the sensitive and selective determination of NTs which have been described in the past two decades with a distinctive focus on extremely innovative attribut,es introduced by nanotechnology.

\section{KEYWORDS:}

Nanomaterials, electrochemistry, biosensors, neurotransmitters, point-ofcare, $\underline{\text { diagnostics }}$ 


\section{Introduction}

The brain is the most complex organ in a vertebrate's body and presents a very challenging and exciting environment for sensing of small molecules called neurotransmitters (NTs).(1) Neurotransmitters are endogenous molecules which act as chemical messengers and are involved in the neurotransmission between neurons. They play a vital role in brain development, learning, memory, mood, sleep, consciousness, blood pressure, and heart rate.(2-6) Neurotransmitter disease is an umbrella term that defines disorders arising due to the discrepancy or imbalance in the metabolism, synthesis, and catabolism of neurotransmitters. Diseases such as Alzheimer's, Parkinson's, dementia, epilepsy, Segawa disease, bipolar disorder, autism, and autism spectrum disorder involve an imbalance in the levels of neurotransmitters that affect signal transduction between neurons. $(7,8)$ This imbalance in the level of neurotransmitters may be due to the consumption of alcohol and drugs, poor dietary habits, genetic predisposition, intake of caffeine, or environmental factors.(9) The environmental factors include consumption of heavy metal ions through water, where increased deposits in the body could alter the functioning of neurons.(10)

Neurotransmitter disorders affect people at different age groups such as pediatric and geriatric populations. Current techniques to detect neurotransmitter-based disorders include MRI imaging, CT scans, and collection of cerebrospinal fluid (CSF) for the measurement of homovanillic acid, neopterin, and BH4 (tetrahydrobiopterin) levels. All these methods include expensive instrumentation, painful surgical procedures, and long analysis time, and also require trained personnel to operate and analyze the results.(11-15) In the context of detection and quantification of neurotransmitters, several strategies have been employed. The conventional method for the detection and quantification of neurotransmitters is microdialysis, $(16)$ which is carried out by using a semipermeable probe that is injected into the brain.(17) Apart from being invasive, another disadvantage of microdialysis is that the technique exhibits low temporal resolution, $(1,18)$ and the withdrawal of the sample for analysis can cause severe damage to the brain tissue.(19,20) Alternately, neurotransmitters can be suffused through the probe for chemical analysis by techniques such as liquid chromatography that is again interfaced with mass spectroscopy or fluorescence spectroscopy for the detection. $(8,18,21)$ Other detection strategies include electrophoresis $\underline{(22)}$ and optical methods such as photoluminescence, $(23)$ colorimetric analysis, $(24)$ etc. However, all these methods are limited by tedious analysis processes, long time requirement for analysis, and requirement for skilled personnel to handle the high cost capital equipment.(25) Development of strategies with improved accuracy and sensitivity of measurement of low levels of these neurotransmitters will enable early diagnosis and treatment of neurotransmitter disorders, thereby reducing the risk of irreversible complications. Biosensors have been developed for quantification of these neurotransmitters as biomarkers of brain disorders. 
There are three main types of biosensors depending on the type of transduction element used. They are colorimetric, electrochemical, and piezoelectric sensors. Among these, electrochemical sensors have demonstrated their ability to quantify and qualify the neurotransmitters. They can also overcome the shortcomings of conventional techniques through the employment of nanointerfaces. $(2,26)$ Here in this review, we focus on the detection of neurotransmitters using nanointerface-modified electrodes. The advantages of the detection of neurotransmitters using nanomaterials include excellent spatial resolution, high sensitivity, improved mass transport, quick electron transfer, negligible surface fouling, and low background current.(2,26-28) The nanointerfaced sensors display improved sensor characteristics such as rapid response, low sample volume, and high sensitivity. Single nanomaterials have earlier been explored as interface materials. Recently, combinations of these nanomaterials have been used as hybrid interfaces $(28-30)$ that serve to further enhance the sensing properties in terms of sensitivity and help to increase the dynamic range of detection. These parameters become invaluable in the field of clinical diagnostics. This review covers the details of the functionality of neurotransmitters to fuel the research appetite of readers focusing on the research and development of brain disorders. Recent advances in nanomaterials that are used to develop electrodes with metallic and polymeric substrates are critically reviewed, and their performance in terms of limit of detection, response time, and stability are discussed. The underlying mechanism and nanomaterial properties responsible for high sensitivity and fast response are highlighted. Both electrochemical and enzyme immobilized biosensors are critically reviewed for a range of biological fluids. Graphene, single- and multiwalled carbon nanotubes, metal oxide nanoparticles, and metallic nanoparticles are predominantly used in the fabrication of sensors for NTs and therefore are the focus for this review. High aspect ratio, large surface area for catalytic activity, and a high chemical stability once bonded with substrate material are characteristics sought and which make nanostructures promising candidates for development of novel sensors for NTs.

\section{Typical Neurotransmission Process ARTICLE SECTIONS}

Neurotransmission is a process of release of neurotransmitters from the axonal terminal of one neuron (presynaptic neuron) to the dendritic terminal of another neuron (postsynaptic neuron).(31) A similar process occurs in the opposite direction during retrograde neurotransmission.(32) This means that the dendrites in the postsynaptic neuron release the retrograde neurotransmitters to the axonal terminal of presynaptic neurons.(33) The process of neurotransmission mainly depends on the amount of neurotransmitter, release rate of neurotransmitter, connection between presynaptic neurotransmitter and postsynaptic neurotransmitter receptor, as well as its activity.(34)Figure 1 shows the process of neurotransmission between presynaptic and postsynaptic neurons. When the action potential reaches the axonal terminal of presynaptic neuron (step 1), it triggers the opening of the voltage-gated $\mathrm{Ca}^{2+}$ channels 
(step 2). Through these channels, $\mathrm{Ca}^{2+}$ enters the presynaptic neuron and activates the synaptic vesicles to release the neurotransmitters (step 3).(6,35) These neurotransmitters are released through the pores into the synaptic cleft at the gap junction between the presynaptic and postsynaptic neurons (step 4).(31,36) Neurotransmitters bind to the neuroreceptors on dendrites of postsynaptic neurons (step 5) and enter to trigger the signal in the postsynaptic neuron (step 6). The binding of the neurotransmitter will influence the postsynaptic neuron by either inducing excitation or inhibition of the action potential.

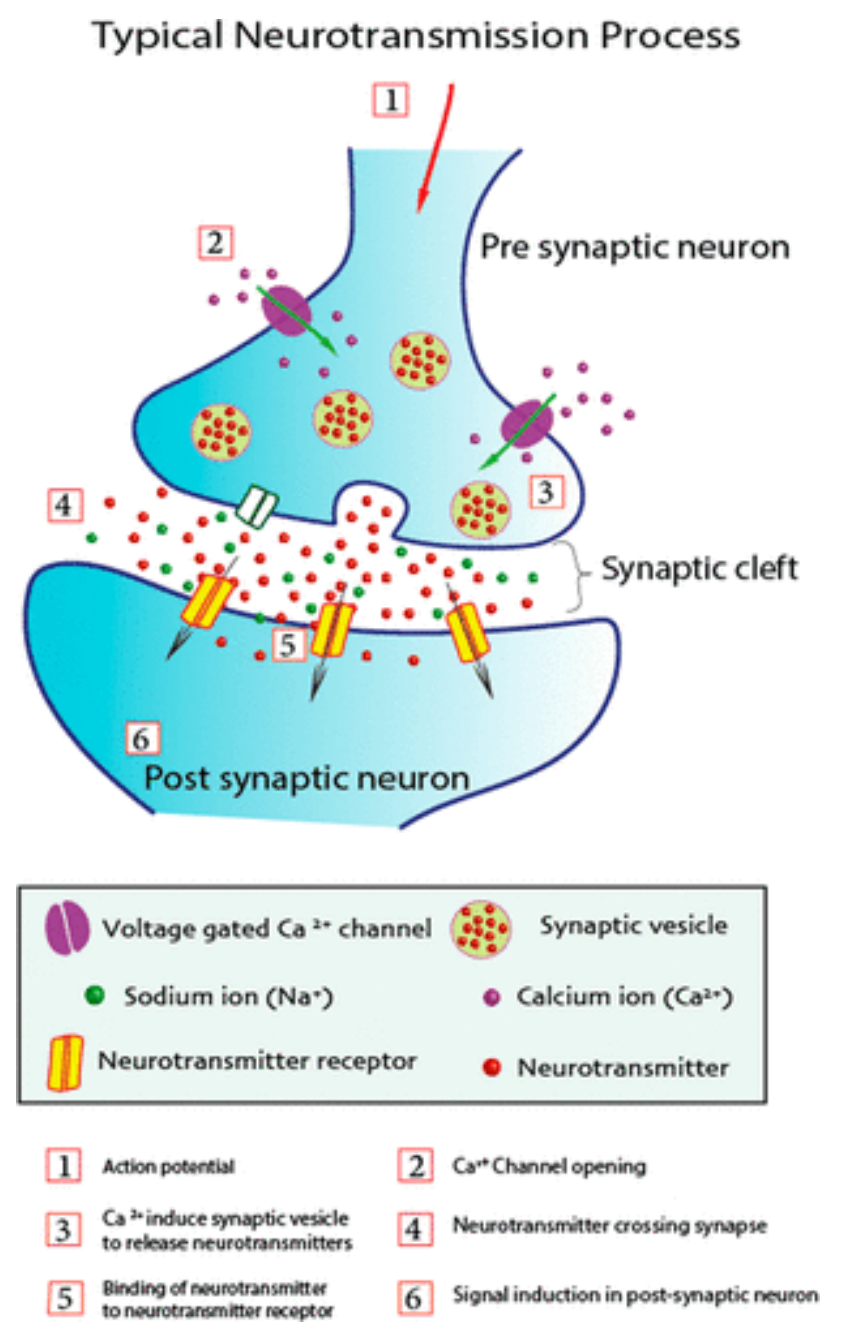

Figure 1. Schematic representation of the neurotransmission process occurring between presynaptic and postsynaptic neurons.

\section{Neurotransmitters and Their Electrochemical Detection Strategie}

Even though there are more than 100 messenger molecules identified in the neurotransmission process, NTs can be classified into three major types based on their structure, mode of action, and physiological function.(37) The NTs can be classified as either direct or indirect based on their mode of action and as excitatory or inhibitory based on their physiological function.(5) However, in this review, we have focused on 
NT classes based on their chemical structure and detailed the different electrochemical strategies reported for their detection. On the basis of the chemical composition and structure, NTs are classified as amines, amino acids, and gaseous substances.(5,38) Dopamine, serotonin, histamine, epinephrine, norepinephrine, and acetylcholine fall under biogenic amine category, whereas glutamate, gamma aminobutyric acid (GABA), tyrosine, and glycine come under amino acid-based NTs. Finally, molecular and gaseous NTs found in the biological system are nitric oxide and $\mathrm{H}_{2} \mathrm{~S}$.(9)Scheme 1 shows the classification of the NTs based on their structural composition and the list of diseases associated with deregulation of these NTs. $(38,37,39)$ Several research groups have published review articles on the electrochemical strategies for the detection of NTs $(38,40-47)$ using biological materials and in vivo methods.(48) Though all these articles give some information on the detection methods of NTs, in this review we have highlighted amine-based NTs and their electrochemical detection methods that have been developed to date employing nanomaterial interfaces. Biogenic amine NTs play a key role in regulating important physiological processes spanning sleep to regulation of blood pressure. Their deregulation contributes to the onset of various neurological disorders. Recently, a link between acetylcholine dysregulation and COVID-19 has also been postulated.(49) These indicate the importance of monitoring the levels of these important classes of NTs which forms the focus of this review. A section on simultaneous detection of these NTs has also been included that could be more relevant for clinical diagnosis.

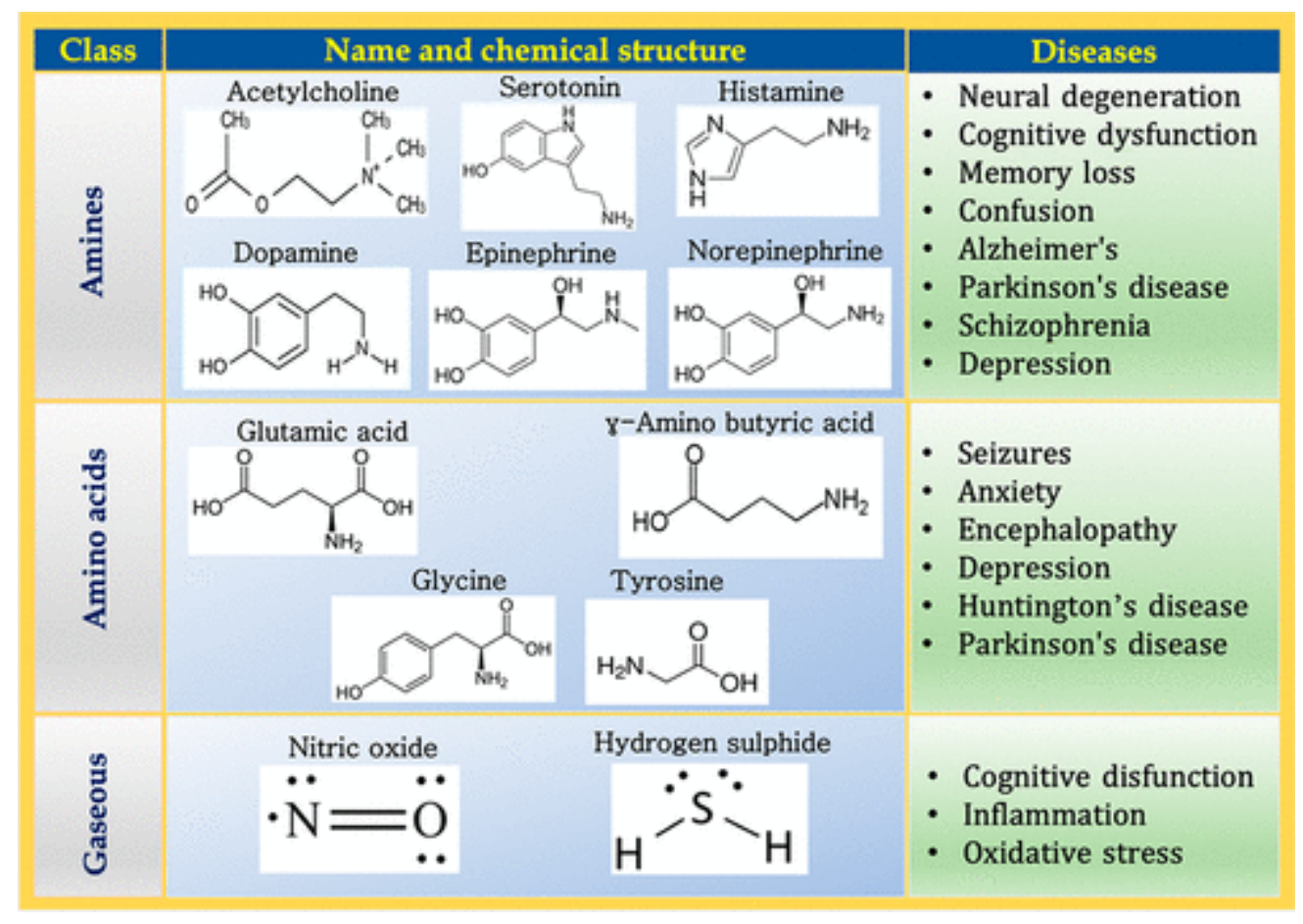

Scheme 1. Classification of Neurotransmitters Based on Chemical Composition and Diseases Associated with Their Deregulation $\underline{(38,37,39)}$ 


\section{Acetylcholine (ACh)}

Acetylcholine (ACh) is the first identified neurotransmitter in the peripheral and central nervous system. It plays a vital role in memory, learning, emotions, and movement and also acts as a messenger from nerves to the muscles.(50) A decrease in the levels of ACh leads to several neurological disorders such as Alzheimer's disease, dementia, schizophrenia, Myasthenia Gravis, and Parkinson's disease etc., while increased levels of ACh leads to a reduction in heart rate.(50) Quantification of acetylcholine levels plays a crucial role in clinical applications to understand disease pathologies associated with cholinergic transmission. The normal range of ACh in human blood lies between 0.2 and $1.31 \mu \mathrm{mol} / \mathrm{L}$.(51) A nonenzymatic sensor was developed using glassy carbon electrode (GCE) modified with carbon dot-decorated nickel-aluminum layered double hydroxides (NiAl-LDH) which displayed a high sensitivity toward ACh.(52) Electrocatalytic oxidation of the enzyme acetylcholine esterase (AChE) was performed on a carbon paste electrode modified with nickel oxide lichen-like nanostructures and used as the working electrode for the sensing of ACh.(53) The lichen-like nanostructures increase the electroactive area, which contributes to the noted improved sensing performance.

Many research groups have worked on the simultaneous detection of acetylcholine and choline $(\mathrm{Ch})$, which is the precursor for the synthesis of ACh using acetylcholine esterase and choline oxidase (ChOx) enzymes, respectively. Sequential biochemical reactions of the working mechanism of the AChE/ChOx bienzyme sensor are shown in $\underline{\text { Scheme } 2 .}$

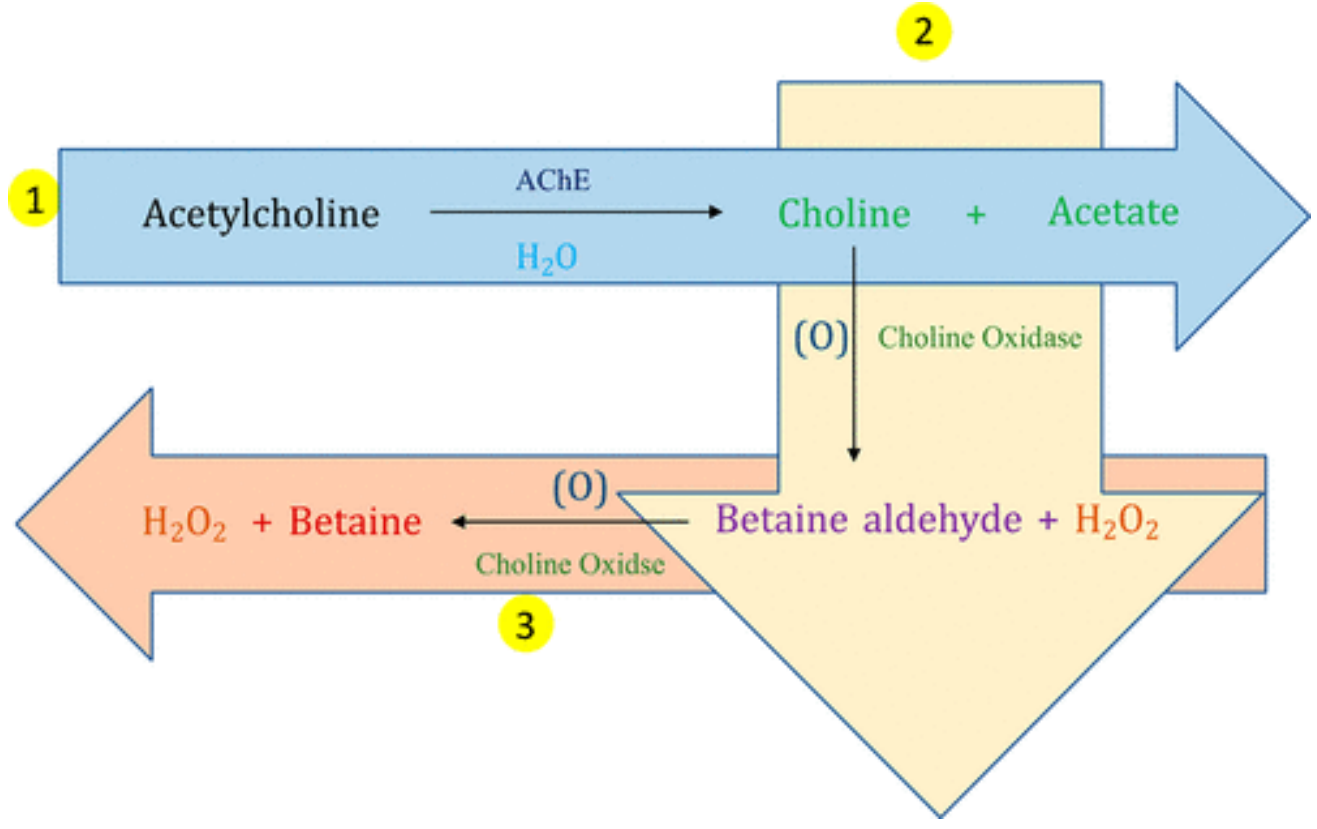

Scheme 2. Reactions Mediated by Acetylcholine Esterase and Choline Oxidase

Acetylcholine will be acted upon by $\mathrm{AChE}$ and $\mathrm{ChOx}$ in successive steps in the presence of oxygen, resulting in the formation of $\mathrm{H}_{2} \mathrm{O}_{2}$. Depending on the magnitude of the oxidation current of $\mathrm{H}_{2} \mathrm{O}_{2}$, the acetylcholine and choline levels can be quantified. In a 
typical bienzyme sensor, graphene oxide ionic liquid (GO-IL) was drop-casted on GCE surface and dried to form a stable gel-like structure. Acetylcholine esterase and choline oxidase were adsorbed on the surface of the GCE/GO-IL electrode and used as the working electrode for the simultaneous detection of ACh and Ch.(54) In another report, $\mathrm{AChE}$ and $\mathrm{ChO}$ immobilized on gold electrode modified with platinum nanoparticles and metal organic framework were used for the simultaneous detection of acetylcholine and choline in serum.(55) An even better detection limit was reported for AChE-ChOx cross-linked with glutaraldehyde and bovine serum albumin on polypyrrolepolynvinyln sulfonate (PPy-PVS) film.(56) In an interesting strategy, a platinum electrode was modified with thiolated silica gel containing multiwalled carbon nanotubes and ChOx followed by incorporation of gold nanoparticles (AuNps). On the top of this layer, diallyl dimethylammonium chloride (PDDA) and AChE were coated in a layer-by-layer assembly to optimize the AChE loading.(57) A shorter response time of $4 \mathrm{~s}$ was reported when a nanocomposite of carboxylated multiwalled carbon nanotubes and zirconium nanoparticles was employed as depicted in Figure 2B.(29)

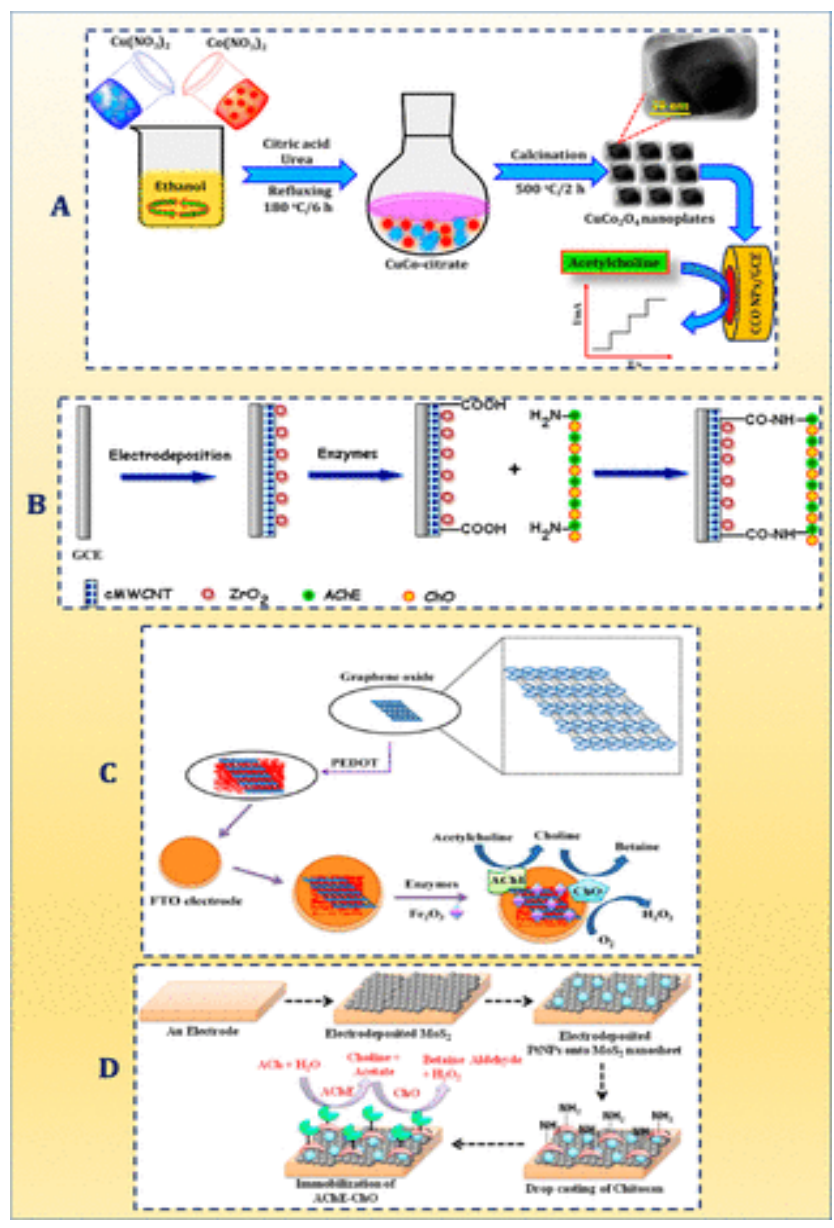

Figure 2. Preparation methods of different nanomaterials used for ACh detection (A) copper cobaltite.(59) Reprinted with permission from ref (59). Copyright 2019 American Chemical Society. (B) Composite of multiwalled carbon nanotubes and zirconium oxide nanoparticles.(29) Reprinted from ref (29). Copyright 2012, with permission from Elsevier. (C) Reduced graphene oxide with iron oxide 
nanoparticles.(60) Reprinted from ref (60). Copyright 2017 with permission from Elsevier. (D) Palladium and molybdenum disulfide nanoparticles.(61) Reprinted from ref (61). Copyright 2019, with permission from Elsevier.

In a study employing a gold electrode, gold coated iron oxide nanoparticles (Fe@Au Nps) were electrodeposited on the gold surface, which was then dipped into chitosan solution, dried, and immersed in glutaraldehyde for cross-linking the polymer. The AChE/ChO enzyme mix was then coimmobilized on this layer, and this electrode was used as the working electrode. This sensor exhibited good stability over a period of 3 months. $(58)$

Apart from the nanointerfaces, different types of electrode materials have also been explored for detection of $\mathrm{ACh}$ and $\mathrm{Ch}$. Fluorine doped tin oxide electrode was modified with a nanocomposite comprising of reduced graphene oxide (rGO), poly $(3,4-$ ethylenedioxythiophene) (PEDOT) and $\mathrm{Fe}_{2} \mathrm{O}_{3}$ nanoparticles. $\mathrm{AChE}$ and ChOx enzymes were immobilized on this modified electrode (Figure 2C) for sensing of acetylcholine in human serum.(60) Two-dimensional molybdenum disulfide nanoparticles synthesized using a ball milling method were electrodeposited on a gold electrode. Palladium nanoparticles were also electrodeposited on the modified gold electrode $\left(\mathrm{MoS}_{2}\right.$-Au-ET). Co-immobilization of $\mathrm{AChE}$ and $\mathrm{ChOx}$ was carried out after dropcasting chitosan on Pd-MoS $-\mathrm{Au}-\mathrm{ET}$ (Figure 2D). This sensor was stable for about 90 days and exhibited a low detection limit of $1 \mathrm{nM}$, which is superior than earlier reports for the quantification of these analytes.(61) Highly porous gold (HPG) was electroplated over a platinum electrode followed by immobilization of AChE using electrodeposition, and this electrode was used for sensing studies.(50) Ion selective electrodes made up of poly(3,4-ethylenedioxythiophene)/poly(styrenesulfonate) (PEDOT/PSS) were also used for electrochemical detection of ACh.(62) Screen printed electrodes modified with magnetic core-shell manganese ferrite nanoparticles were successfully employed for ACh detection in serum samples, which represents the next dimension in electrochemical sensing.(63)Figure 2A depicts the scheme for the synthesis of copper cobaltite nanoparticles (CCO NP) synthesized using copper nitrate and cobalt nitrate as precursors. CCO NP were drop-casted on GCE electrode and successfully employed for the detection of $\mathrm{ACh}$ with a wide linear range from $0.2 \mu \mathrm{M}$ to $3500 \mu \mathrm{M}$. The sensor reported a short response time of $4 \mathrm{~s}$.(59) This sensor might be a promising candidate for $\mathrm{ACh}$ quantification in clinical samples. A hybrid nanointerface of MWCNTs, $\mathrm{MnO}_{2}$, and rGO was employed recently for the detection of ACh in serum samples.(64)

From these studies, it can be concluded that metallic and carbon nanostructures demonstrate promising results for the detection of $\mathrm{ACh}$ and $\mathrm{Ch}$. The difference in the linear range and sensitivity between the different nanostructures could be attributed to the differences in the surface area-to-volume ratio, dimensions of the particles, surface coverage, and nature of the nanointerface employed. Graphene enabled an extremely low limit of detection $(0.885 \mathrm{nmol} / \mathrm{L})$ for $\mathrm{ACh}$ in $90 \mathrm{~s}$. Metallic nanoparticle (gold, 
platinum, and copper cobaltite) based composites provided reliable results with limit of detection in nanomolar concentrations within 3-4 s. Gold used as the electrode substrate material or as interface coating provided good reliable performance over three to four months. Beside metallic surfaces, polymeric substrates (e.g., poly pyrrole-polynvinyl sulfonate (PPy-PVS) film) also provided a limit of detection at nanoscale concentrations; however, their response time was long (200 s) compared to graphene (90 s) and metallic nanoparticles (3-4 s). Drop casting, spin coating, and electroplating have mostly been used for the fabrication of ACh sensors. For large area electronics, screen printing is a suitable technique and commercially used in the printed and flexible electronics industry.(49) For ACh sensors, screen printing has been shown to be able to provide a relatively low limit of detection $(20 \mathrm{nM})$. Metallic nanostructures have emerged as preferential candidates for ACh detection with the combination of a fast response time (3-4 s), low limit of detection (in nanorange), and excellent stability (34 months).

\section{Glutamate}

Glutamate is the precursor of GABA and plays a vital role in the pathophysiology of neurotransmitter disorders.(65) It is a nonessential amino acid and acts as an excitatory neurotransmitter.(66) It is also used as a food additive and leads to "Chinese-restaurant syndrome" presently referred to as the monosodium glutamate (MSG) syndrome.(67) Glutamate is distributed throughout the central nervous system and mediates several functions in the brain that involve learning and memory. It also plays a major role in the formation of synapses. The concentration of glutamate in the nervous system is large (5-15 mmol kg-1) when compared to the extracellular fluids where the concentration is in the micromolar range. Therefore, minute changes in the concentration of the glutamate levels impact the nervous system and lead to disturbances in the normal signaling pathways.(68) Dysregulated release of glutamate leads to several neurological disorders such as Alzheimer's disease, epilepsy, Parkinson's disease, and ischemia.(5) In the context of quantification of glutamate, the use of the glutamate oxidase enzyme has been extensively investigated. The reaction scheme involving glutamate oxidase on glutamate is shown in $\underline{\text { Scheme } 3}$.

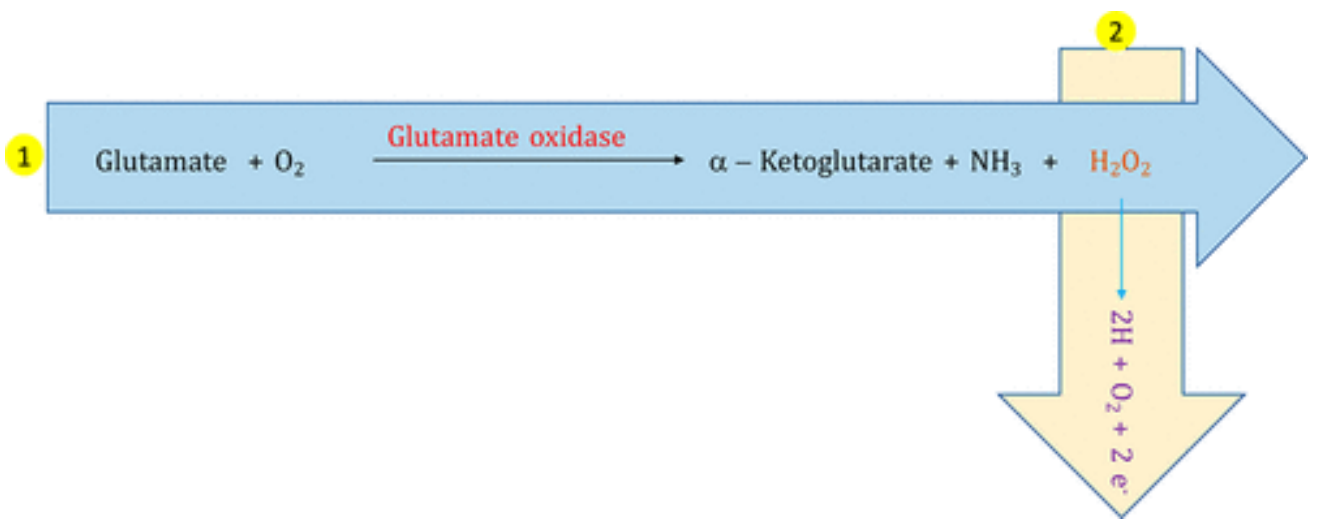

Scheme 3. Reaction Scheme Involving the Transformation of Glutamate to $\alpha$ Ketoglutarate by Glutamate Oxidase 
Rahman et al. in 2005 reported the measurements of glutamate in vivo. The work employed the conducting polymer 5, 2':5',2"-terthiophene-3'-carboxylic acid nanoparticles deposited on a platinum microelectrode. The glutamate levels were measured using cyclic voltammetry at high scan rates. The nanostructured polymer layer resulted in high surface area available for enzyme immobilization by making covalent bonding. This enabled a low limit of detection $(0.1 \mu \mathrm{M})$ with a $10 \mathrm{~s}$ response time. This developed sensor was successfully employed for the measurement of extracellular glutamate levels in the corneal section of rat brain.(69) In a similar strategy, poly- $o$-phenylenediamine modified platinum wire microelectrode was utilized for the in vivo quantification of glutamate levels in brain tissue. Poly-ophenylenediamine was electrodeposited on the Pt wire microelectrode, which served as a permselective membrane. Later, the enzyme glutamate oxidase, which is selective for glutamate, was entrapped in a chitosan polymer matrix, and ascorbate oxidase immobilized in the outer layer was used as the working electrode for quantification of glutamate. The sensor performance was evaluated using an amperometry technique for the detection of glutamate levels in vivo with a quick response of $2 \mathrm{~s}$, and the achieved limit of detection was nanomolar.(20) Similarly, platinum microelectrode coated with poly(phenylenediamine) (PPD) was investigated for glutamate detection in rat brain and displayed stability for a period of 150 days.(4) Among the various types of nanointerfaces, carbon-based nanostructures have been extensively employed for glutamate detection. Single-walled carbon nanotubes functionalized with ferrocene were reported for the detection of L-glutamate with a detection limit of $1 \mu \mathrm{M} .(70) \mathrm{A}$ carbon fiber microelectrode coated with nafion and carbon nanotubes was employed for monitoring glutamate release in rat brain.(71) Multiwalled carbon nanotubes and gold nanoparticles were electrodeposited over a chitosan-coated Au electrode followed by immobilization of glutamate oxidase. The sensor was employed successfully for glutamate analysis in serum samples collected from epilepsy patients.(72) Another approach employed a pencil graphite electrode modified with $\mathrm{ZnO}$ nanorods and polypyrrole followed by immobilization of glutamate oxidase, which was found to be effective in the detection of glutamate for 100 measurements.(73) Detection of glutamate in a hypoxic environment has also gained significance as it is now established that glutamate levels are modulated in response to hypoxia. A hybrid nanointerface of ceria and titanium nanoparticles was investigated for glutamate analysis in cerebrospinal fluid as well as in the brain of Sprague-Dawley rats. In this study, a platinum microelectrode was modified with chitosan-ceria-titania nanocomposite, and then the enzyme glutamate oxidase was immobilized as shown in Figure $3 \mathrm{~A}$. This sensor exhibited a response time of $2 \mathrm{~s}$ in oxygenated conditions and $5 \mathrm{~s}$ in deoxygenated conditions.(74) Electrochemiluminescence sensor using a hybrid interface of carboxylated multiwalled carbon nanotubes and graphene was also reported for glutamate sensing (Figure 3B).(75) 


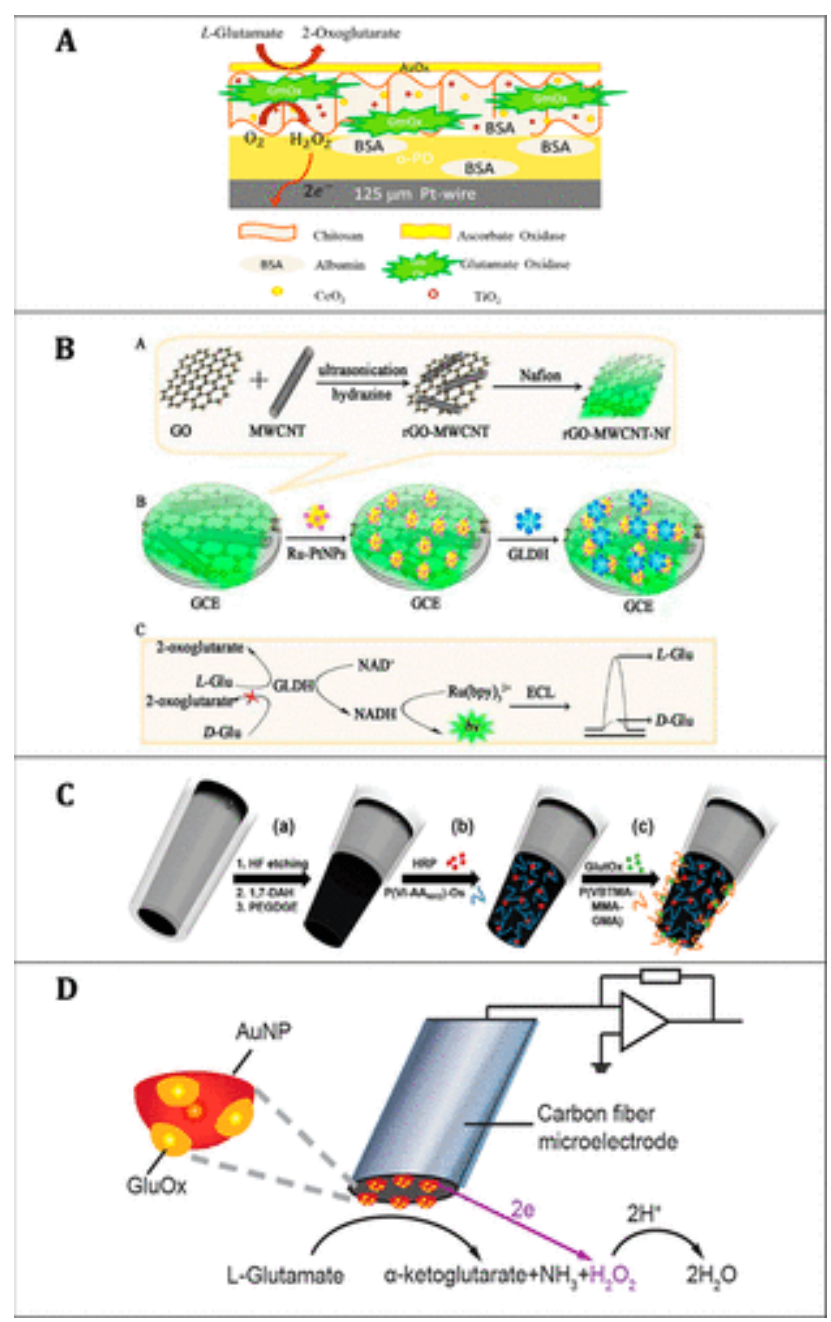

Figure 3. Utilization of (A) hybrid interface consisting of ceria and titanium nanoparticles.(74) Reprinted from ref (74). Copyright 2014 with permission from Elsevier. (B) Multiwalled carbon nanotubes with reduced graphene oxide.(75) Republished with permission of Royal Society of Chemistry from ref (75) permission conveyed through Copyright Clearance Center, Inc. (C) Carbon nanoelectrodes.(76) Republished with permission of Royal Society of Chemistry, from ref (76), permission conveyed through Copyright Clearance Center, Inc. (D) Carbon fiber microelectrode.(77) Reprinted with permission from ref (77). Copyright 2019 American Chemical Society, for the successful detection of glutamate.

As shown in Figure $3 \mathrm{C}$, enzyme-modified carbon nanoelectrodes were used for the intracellular detection of glutamate in astrocytes.(76) The ultrasmall dimensions and tapered tip of the electrode enables penetration through the cell membrane with no permanent damage to the cells. Similarly, a carbon microfiber electrode coated with gold nanoparticles and glutamate oxidase was reported for the amperometric detection of glutamate levels based on $\mathrm{H}_{2} \mathrm{O}_{2}$ release(77) (Figure 3D). Using a similar strategy, a carbon fiber electrode modified with Prussian blue (PB), poly- $o$-phenylenediamine (poPD), and polyethylenimine (PEI) was cross-linked with glutamate oxidase, and glutaraldehyde was successfully employed for the determination of glutamate levels.(78) 
Gholizadeh et al. have reported the fabrication of a glutamate biosensor based on vertically aligned carbon nanotubes forming a nanoelectrode array (VACNT-NEA) developed using photolithography. This sensor exhibited two linear ranges of 0.1-20 $\mu \mathrm{M}$ and $20-300 \mu \mathrm{M}$ with superior sensitivity.(79) Glutamate displays a high affinity toward $\mathrm{H}_{2} \mathrm{O}_{2}$. On the basis of this property, Isoaho et al. have reported a glutamate biosensor using carbon nanofibers grown on amorphous carbon. Glutamate oxidase was then immobilized using $N$-(3-(dimethylamino)propyl)- $N$ '-ethylcarbodiimide and $N$ hydroxysuccinimide (EDC-NHS) cross-linking. This sensor reported a rapid response of $0.5 \mathrm{~s}$ and was successfully employed for glutamate detection in glial cells. $\underline{(80)}$

Amperometric detection of glutamate levels in brain terminals called synaptosomes as well as in blood has been reported.(81) A disposable biosensor based on platinum nanoparticles electrodeposited on gold nanowire for simultaneous detection of glutamate and $\mathrm{H}_{2} \mathrm{O}_{2}$ has been reported by Mamum Jamal et al.(82) A human microelectrode array prototype was successfully used for real time monitoring of glutamate levels in Rhesus monkeys.(83)

There are several research groups actively involved in the development of sensor devices that use ultramicro- or nanoelectrodes for in vivo detection of glutamate. Hybrid sensors composed of carbon nanostructures (single walled and multiwalled nanotubes and graphene) are predominantly reported in the literature for glutamate detection. Conducting polymer-based nanostructures also have demonstrated excellent limit of detection $(0.2-1.6 \mu \mathrm{M})$ and fast response time (1-2 s). There has been an exponential increase in the demand for glutamate sensing systems because of their emerging clinical importance in diagnosis and understanding neuronal diseases, and therefore, opportunities still exist toward development of glutamate sensors with superior sensing performance as point-of-care diagnostic devices.

\section{Dopamine}

Dopamine is a catecholamine, and its levels are related to the biodegradation of tyrosine.(84) As a neuromodulator, it plays a crucial role in learning, sleep, cognition, neuronal plasticity, and movement.(85) Dopamine is classified under monoamines and acts as a precursor of adrenaline and noradrenaline. Its abnormal levels are related to several neurotransmitter disorders, namely, Tourette syndrome, Alzheimer's disease, psychosis, Parkinson's disease, Huntington's disease, senile dementia, and schizophrenia. $(86,87)$ Dopamine has been reported as a clinical biomarker in many neurotransmitter disorders, and hence the measurement of dopamine levels is of great clinical importance.(23) There have been several reports on electrochemical dopamine sensors employing nanointerfaces. A carbon fiber microelectrode (CFME) was employed for the in vivo detection of dopamine. The CFME was modified with $\mathrm{TiO}_{2} / \mathrm{CeO}_{2}$ nanocomposite dispersed in chitosan, and then the enzyme tyrosinase was electrostatically coupled with the amino group of chitosan. The sensor was successfully employed for monitoring dopamine levels in rat brain for a period of $2 \mathrm{~h}$.(88) As the diameter of the microelectrode was around $100 \mu \mathrm{m}$, in vivo detection was possible with 
this implantable enzyme-based biosensor. However, the stability of the sensor reduced over time, and $87 \%$ loss of function was experienced after 1 week. This was improved by depositing ceria-based metal oxides, and the biosensor response was increased to 90\% in 1 week. In another study, microelectrodes made up of carbon nanotube modified yarn (CNTYM) were reported for the detection of dopamine levels. The laser-treated CNTYM was employed as the working electrode (CNTYME) using a fast scan cyclic voltammetry technique. The unmodified CNTYME showed a low background current of $86 \pm 6 \mathrm{nA}$, while the laser-treated CNTYME exhibited a higher background current of $161 \pm 18 \mathrm{nA}$, which represents a $1.9 \pm 0.2$ fold improvement in the analytical performance of the laser-treated CNTYME for the detection of dopamine levels with a limit of detection of $13 \pm 2 \mathrm{nM}$. (89) Nonenzymatic sensors have also been explored for quantification of dopamine because enzymatic sensors pose challenges in immobilization of the enzyme without loss of function and are more expensive. Bimetallic nanospheres comprised of a palladium-silver alloy coated with polypyrrole (Pa-Ag NSps-PPy) were employed for nonenzymatic electrochemical detection of dopamine levels.(90) Bowling-alley-like hollow arrays made up of self-assembled AuAg nanocomposites were functionalized with L-cysteine (Cys-Au@ Ag BMNPs). This composite was coated on a glassy carbon electrode and used as the working electrode for nonenzymatic detection of dopamine (Figure 4A).(91) Polypyrrole-silverpolyvinylpyrrolidone has also been employed for nonenzymatic detection of dopamine.(92) Recently, a wearable electrochemical sensor was developed by Qing et al. using poly(vinylalanine) nanofibers and polypyrrole nanofiber networks (Figure 4B). The sensor was based on a field effective transistor-based electrochemical mechanism.(93) One of the challenges in nonenzymatic sensors is ensuring their selectivity and specificity toward dopamine. In this context, the enzymatic sensors exhibit superiority over their nonenzymatic counterparts; however, as with any biosensor, stability is still challenging for biosensors due to loss of enzymes and coated layer interface. Therefore, the repeatability is only achieved for a few measurements and up to few days.

Fabrication of nanoscale sensors is challenging, and nonuniformity in the fabricated sensor sensing sites is common. Therefore, investigations focused primarily on the nanoscale fabrication technique are crucial. Screen printing allows fabrication of multimaterial sensors for simultaneous detection of multiple species. In a study, screenprinted carbon electrode modified with poly(3,4-ethylenedioxythiophene)-PEDOT and graphene oxide ink was employed for the detection of dopamine along with other molecules such as uric acid, ascorbic acid, and nitrite encountered in the biological system (Figure 4C).(94) For dopamine, the achieved limit of detection for screen printed electrode was $30 \mathrm{nM}$, which is comparable to sensor electrodes fabricated through direct drop-casted or electrospinning methods. Core-shell nanostructures comprising multiwalled carbon nanotubes and graphene oxide nanoribbons have also been successfully implemented for the detection of dopamine along with ascorbic acid and uric acid.(95) Hollow microspheres fabricated using two different conducting 
polymers, namely, poly(3,4-ethylenedioxythiophene) and poly( $N$-methylpyrrole) were also employed for dopamine detection.(96) A nanoweb made up of 3D carbon nanotubes was used for electrochemical dopamine detection in the range of 1-20 $\mu \mathrm{M}$.(97) In another strategy, methylene blue (MB) was electropolymerized on reduced graphene oxide (EGO) and doped with dopamine. This dopamine-grafted poly MBEGO composite was employed for dopamine detection.(98) Cylindrical-shaped gold nanoelectrode arrays (CAuNE) have been reported for successful electrochemical detection of dopamine levels in SH-SY5Y cells. This human cell line was derived from SK-N-SH cell line and has been widely reported for the expression of dopaminergic markers.(99)

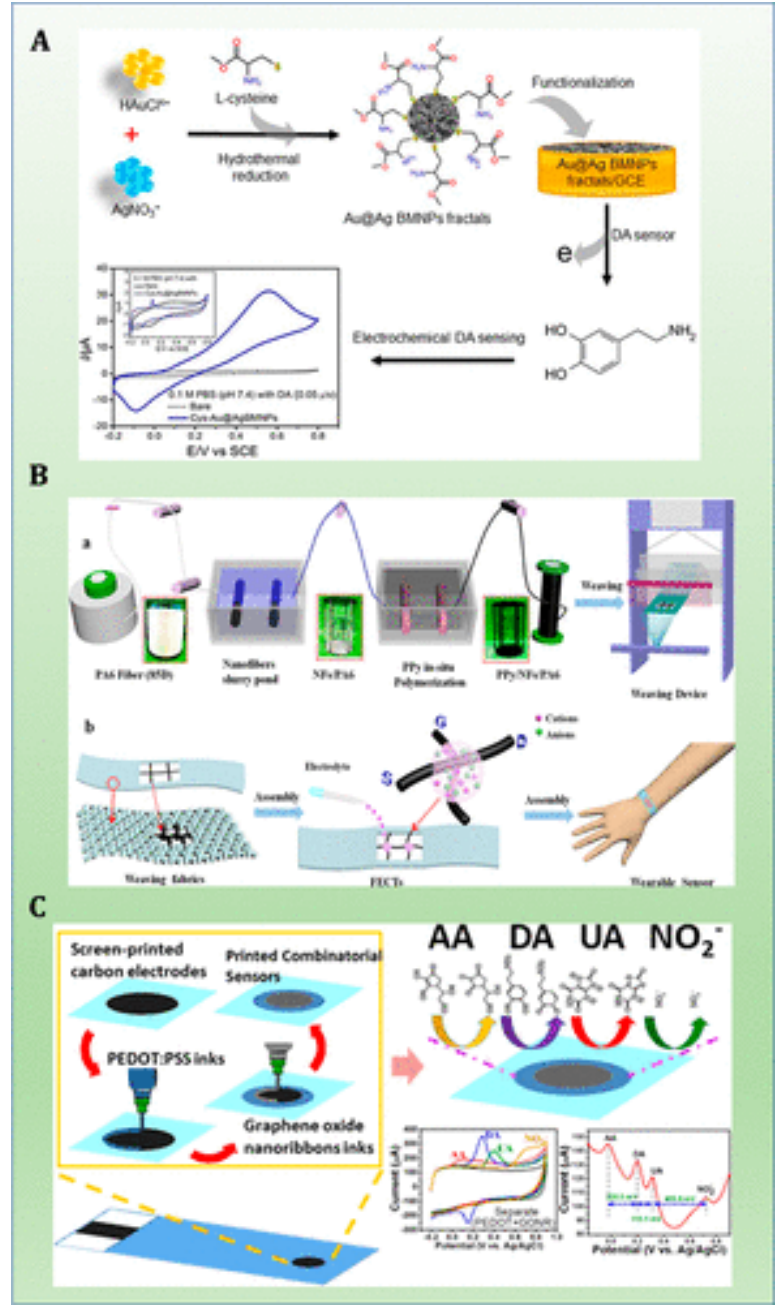

Figure 4. Schematic representations of the preparation strategies of (A) hollow arrays of self-assembled $\mathrm{Au}-\mathrm{Ag}$ nanoparticles $(91)$ Reprinted with permission from ref (91). Copyright 2019 American Chemical Society. (B) Polyaniline-silver- polypyrrole nanofibers.(93) Reprinted with permission from ref (93). Copyright 2019 American Chemical Society. (C) Graphene oxide nanoribbons.(94) Reprinted with permission from ref (94). Copyright 2019 American Chemical Society for electrochemical detection of dopamine. 
In a recent study, $\mathrm{Ag} @ \mathrm{C}$ core-shell nanostructures were synthesized by a hydrothermal method using glucose and $\mathrm{AgNO}_{3}$ as precursors. Then, $\mathrm{HAuCl}_{4}$ was added to the above solution, and the mixture was heated in an oil bath at $100{ }^{\circ} \mathrm{C}$ for about $20 \mathrm{~min}$. The mixture was centrifuged to obtain $\mathrm{Ag} @ \mathrm{C} / \mathrm{Au}$ nanostructures. This composite was mixed with chitosan, drop-casted on the GCE surface, and used as the working electrode postdrying at room temperature (Figure 5D). The $\mathrm{Ag} @ \mathrm{C} / \mathrm{Au}$ composite sensor promoted electrochemical oxidation and reduction of dopamine thereby demonstrating not only high sensitivity but also high selectivity in the presence of other species. This sensor exhibited a low detection limit of $0.21 \mu \mathrm{M}$ and remained stable up to 14 days.(103) Selectivity was also examined by Chang et al. by using a glassy carbon electrode modified with functionalized carbon nanotubes and polyaniline composite for dopamine detection.(104) The composite demonstrated excellent electrochemical reactivity toward the oxidation of dopamine and hence provided very high selectivity in the presence of ascorbic acid and uric acid. In another study, $\mathrm{MoS}_{2}$ nanosheets loaded with bimetallic platinum-nickel nanoparticles have been reported for the simultaneous detection of dopamine and uric acid in urine samples. Because of the high surface area and excellent electrochemical characteristics, the limit of detection PtNi@MoS2/GCE was $0.1 \mu \mathrm{M}$ for dopamine.(105) Other metallic nanoparticles such as tungsten trioxide $\left(\mathrm{WO}_{3}\right),(106)$ iron oxide, $(107)$ and $\mathrm{CuO}$ nanoparticles $(108)$ have also been employed for electrochemical quantification of dopamine. Recently, a comparative study was performed with three different metal oxide (MO) nanoparticles where the electrocatalytic behavior of $\mathrm{NiO}, \mathrm{ZnO}$, and $\mathrm{Fe}_{3} \mathrm{O}_{4}$ nanoparticles was analyzed with and without polyaniline (PANI). This study provided experimental evidence to prove that the PANI-MO composite exhibited better electrocatalytic behavior than the MO alone. PANI with nickel oxide nanoparticles exhibited excellent electrical conductivity and diffusion controlled electrocatalysis of dopamine on the PANI-NiO.(109)Figure 5B shows a two-dimensional copper-based metalorganic framework (Cu-MOF) that was loaded with gold nanoparticles (Au Nps). The resultant conductive poly(xanthurenic acid) p(XA) was successfully employed for electrochemical dopamine sensing.(101) As shown in Figure 5C, a screen-printed electrode modified with graphite and gelatin $\mathrm{x}$ was also investigated for dopamine sensing in the presence of ascorbic acid and uric acid in serum as well as urine samples.(102) Reduced graphene oxide supported with bimetallic core-shell nanoparticles of Au@ Pd was successfully prepared (Figure 5A) and used for dopamine sensing in phosphate buffered saline. $(100)$ Graphene, $(110,111)$ porphyrin-functionalized graphene, $(112)$ grapheneplatinum nanocomposites, $(113)$ and graphene-palladium nanoparticles $\underline{(114)}$ have also been reported for dopamine detection. The lowest limit of detection was achieved at 80 $\mathrm{nM}$, and a $10 \mathrm{~s}$ response time was recorded. The authors demonstrated that graphene composite with well dispersed Pt nanoparticles resulted in a high oxidation rate for dopamine.(113) P-doped graphene was demonstrated to exhibit better loading capacity of metal nanoparticles such as Au Nps.(115) Indium tin oxide (ITO) electrode modified with porous graphene oxide and gold nanoparticles was successfully employed for dopamine sensing.(116) The limit of detection for graphene composite based dopamine 
sensors was $0.2 \mathrm{nM} \underline{(109)}$ to $30 \mathrm{nM} . \underline{(113)}$ The results from these studies indicate that graphene is an excellent candidate for the fabrication of nanosensors for dopamine. A low-cost flexible thin film device using carbon screen printed electrodes was reported recently with a low detection limit of $50 \mathrm{pM}$ toward dopamine.(117) Though a plethora of efforts have been directed toward the development of dopamine sensors, a scope exists for further improvement in the sensing range and an extension of the period of use of these sensors for real-time monitoring of dopamine levels in biological systems to become clinically relevant.

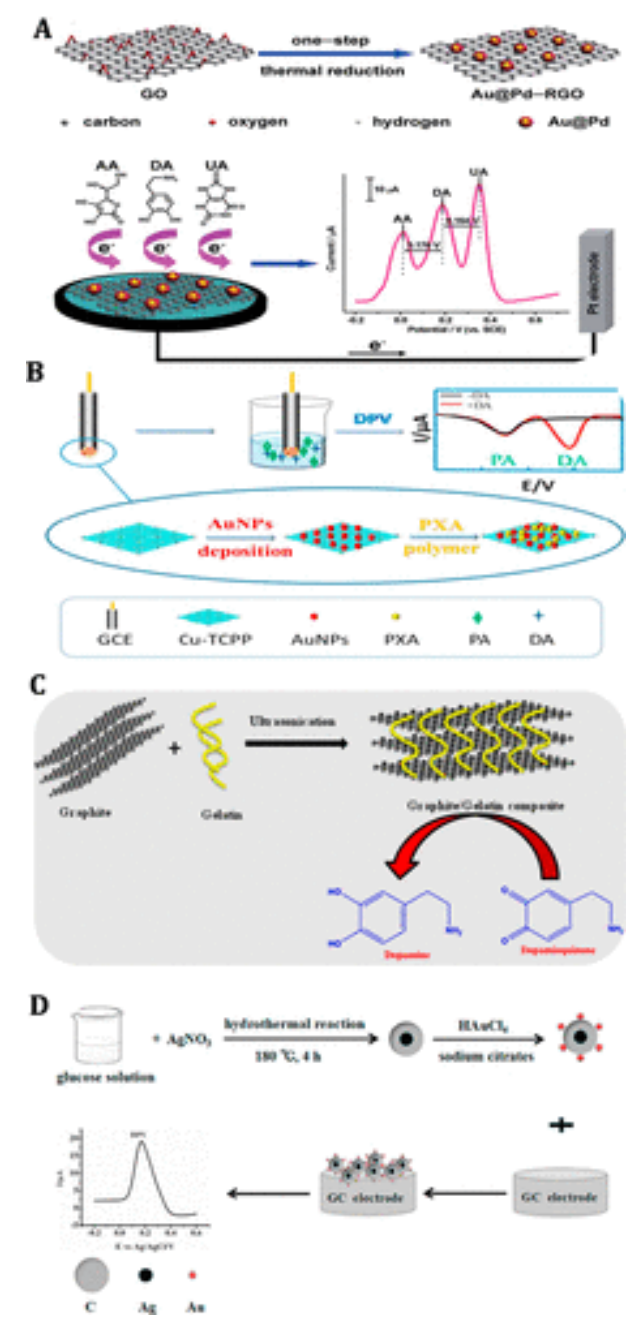

Figure 5. (A) Au@Pd-rGO nanoparticles.(100) Republished with permission of Royal Society of Chemistry, from ref (100), permission conveyed through Copyright Clearance Center, Inc. (B) Cu based MoFs with Au nanoparticles.(101) Reprinted from ref (101). Copyright 2019, with permission from Elsevier. (C) Graphite.(102) Reprinted from ref (102). Copyright 2017 with permission from Elsevier. (D) Ag@C coreshellnano composites.(103) Reprinted from ref (103). Copyright 2019 with permission from Elsevier for dopamine analysis.

\section{Serotonin}

5-Hydroxytryptamine (serotonin, 5-HT) is an important biogenic monoamine neurotransmitter as well as neuromodulator(118) that plays a major role in several 
physical, biological, and psychological processes. Alterations in the secretion levels of serotonin leads to sleep disorders, anxiety, eating disorders, depression, and infantile autism, and also affects muscle contraction and endocrine regulation.(119) Nonenzymatic electrochemical detection of serotonin in both in vitro and in vivo conditions has been reported in the literature. Incorporation of nanointerfaces has been found to be beneficial in extending the detection range and sensitivity, and reducing the response time. Graphene oxide grafted with poly(lactic acid) and palladium nanoparticles (G- $g$-PLA-Pd) coated on glassy carbon electrode was successfully employed for the electrochemical detection of serotonin in a $0.1 \mathrm{M}$ phosphate buffer solution. This sensor exhibited a good linear range from 0.1 to 100 $\mu \mathrm{M}$, and a low limit of detection $(80 \mathrm{nM})$ was achieved. For uniform distribution of GO throughout the organic media, treatment with 1,4-butanediol (BD) resulted in introducing an anchoring $\mathrm{OH}$ site, and the subsequent polymerization was performed to produce GO-g-PLA composites.(120) A glassy carbon electrode was modified with reduced graphene oxide and PANI, then coated with gold nanoparticle-embedded molecularly imprinted polymers (AuNPs@MIPs) (Figure 6A) to enhance the selectivity toward serotonin with a very low limit of detection $(11.7 \mathrm{nmol} / \mathrm{L}) .(121)$ A ternary nanocomposite made up of silver, polypyrrole, and copper oxide $\left(\mathrm{Ag} / \mathrm{PPy} / \mathrm{Cu}_{2} \mathrm{O}\right)$ prepared by a sonochemical method was used for the detection of serotonin in human serum samples with a $0.1 \mathrm{M}$ phosphate buffer solution as a supporting electrolyte(122) (Figure 6C). A three-dimensional structure of gold and polypyrrole nanoparticles (AuNPs@PPyNPs) was electrodeposited on a screen-printed electrode using a multipulse amperometry technique.(123)

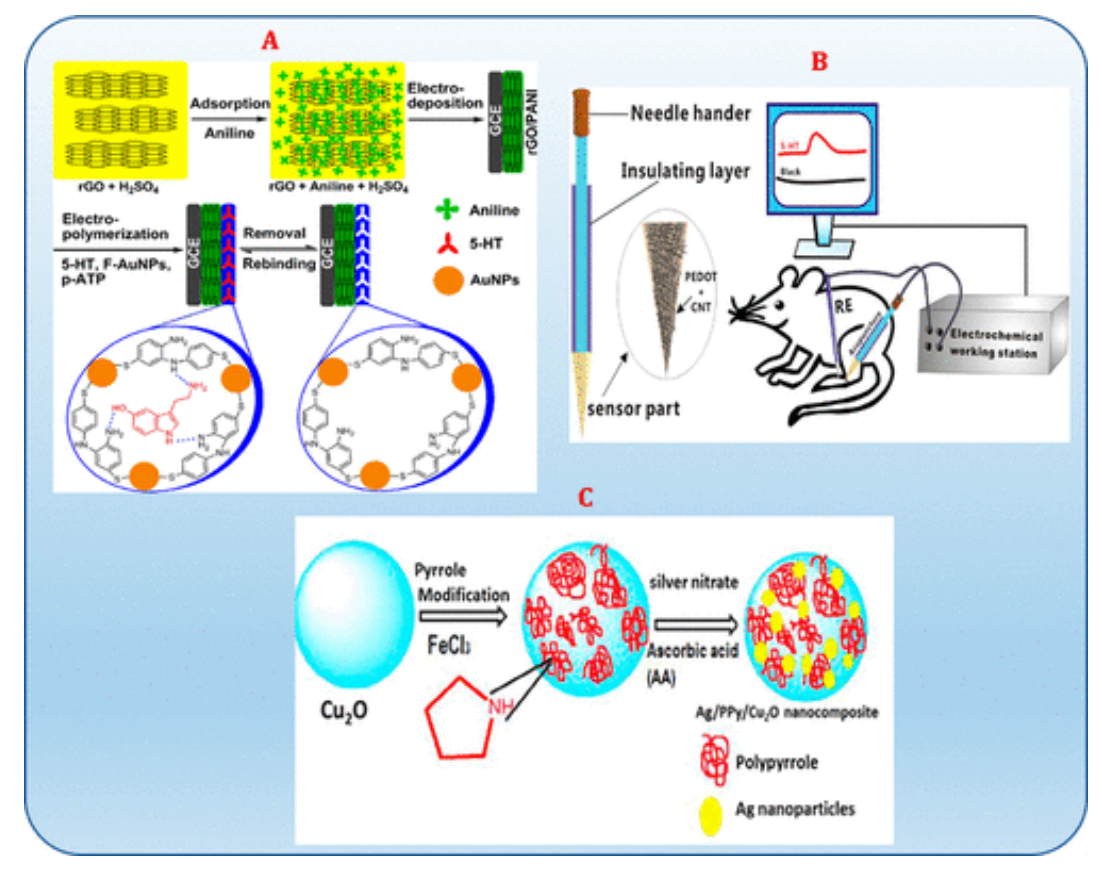

Figure 6. Strategies employed for the detection of serotonin using (A) reduced graphene oxide-Au nano composite.(121) Reprinted from ref (121). Copyright 2014 with permission from Elsevier. (B) Carbon nanotubes.(132) Reprinted from ref (132) Springer Nature and licensed under CC BY 4.0. (C) Ternary nanocomposite 
of Ag/Ppy, $\mathrm{Cu}_{2} \mathrm{O} .(122)$ Reprinted from ref (122). Copyright 2018 with permission from Elsevier.

Electrochemical reduction is a very popular technique for reducing graphene to obtain reduced graphene oxide. Electrochemically synthesized reduced graphene oxide (rGO) followed by electrodeposition of cobalt oxide nanoparticles $\left(\mathrm{Co}_{3} \mathrm{O}_{4}\right)$ was used for fabrication of the sensing element. The RGO- $\mathrm{Co}_{3} \mathrm{O}_{4}$ nanocomposite exhibited a 4.1 fold increased response toward serotonin.(124) This sensor reported the selective detection of serotonin in the presence of potential interferents such as ascorbic acid and dopamine. This work becomes significant because the redox potentials of dopamine and serotonin are very close to each other, and hence there is a possibility of erroneous values obtained from samples containing both molecules. In this context, serotonin detection in the presence of dopamine and ascorbic acid has been reported by using different graphene based composite materials, which includes graphene oxide, thiolated graphene oxides GO-SH and GO-S- $\left(\mathrm{CH}_{2}\right)_{4}-\mathrm{SH}$, graphene oxides reduced using hydrazine and ammonia (RGO1), hydrazine (RGO2), hydroxylamine and ammonia (RGO3). Among all the six types of graphene materials, RGO1 showed a fast response time and better sensitivity in the range of $1-36 \mu \mathrm{M}$.(125) Sadanandhan et al. used a silver patterned reduced graphene oxide (PEDOTNTs/rGO/AgNPs) on a glassy carbon surface for selective detection of serotonin in the presence of tyrosine, ascorbic acid, and dopamine.(126) Similarly, the efficacy of different metal oxide nanoparticles such as $\mathrm{NiO}, \mathrm{ZnO}, \mathrm{Fe}_{3} \mathrm{O}_{4}$, and their conjugated hybrid interface with multiwalled carbon nanotubes (MWCNT) was evaluated. Among all the six interfaces evaluated for the sensing of serotonin in the presence of ascorbic acid and serotonin, MWCNT/NiO exhibited better performance in terms of fast electron transfer and a low limit of detection.(127) A different strategy of competitive adsorption of dopamine was proposed for selective determination of serotonin by Jing-Ming et al.(128) Serotonin was simultaneously detected along with another amino acid levodopa by the differential pulse voltammetry technique using multiwalled carbon nanotubes and chitosan as interface material on the surface of the glassy carbon electrode.(129) In an interesting choice of materials, electro-polymerization of poly(bromocresol green) on a glassy carbon electrode was carried out followed by drop-casting of a $\mathrm{Fe}_{3} \mathrm{O}_{4}-\mathrm{MWCNT}$ composite. This modified electrode was used for the detection of serotonin in the presence of dopamine.(130) Another research group also used an MWCNT-chitosan composite for the modification of a glassy carbon electrode that was successfully employed for the detection of serotonin in the presence of dopamine and ascorbic acid.(131) Real time monitoring of serotonin levels in Wistar rats was performed using an acupuncture needle modified with a PEDOT-CNT composite by $\mathrm{Yu}$-Tao Li et al. (Figure 6B).(132) From these studies, it can be concluded that multiwalled carbon nanotube (MWCNT) composite-based systems exhibit a very low limit of detection $(<1$ $\mathrm{nM}$ ) due to a faster electron transfer process. As MWCNT are chemically stable in composites, the stability of the sensors over multiple measurements and extended periods (e.g., up to several months) should be systematically investigated. Though, the 
progress in the electrochemical detection of serotonin is very impressive, the response times required for the detection of this neurotransmitter reported thus far remains long. Thus, there exists a need for the development of new strategies for rapid detection of this neurotransmitter.

\section{Histamine}

Histidine, a monoamine is the precursor of histamine, which is neither a catecholamine nor an indoleamine. Histamine performs a dual role as a neurotransmitter as well as neuromodulator, and it regulates the release of other neurotransmitters such as $\mathrm{ACh}$, EP (epinephrine), and NEP (nor-epinephrine).(118) It is produced mainly in the mast cells and basophiles and also in tissues and the brain. Histamine produced in the mast cells is called as mast-histamine, and if it is produced in other tissues and brain, then it is referred as non-mast-histamine.(133) Histamine regularly stimulates different types of postsynaptic receptors, which are related to bone marrow, muscles, gastric cells, and brain. It is mainly associated with the immune system to act against pathogens and also plays a role in influencing temperature and blood pressure variations. It is also identified to play a key role in sleep and wakefulness.(134) Neuronal histamine tends to display a diurnal rhythmic pattern with high histamine levels found during the waking period and low histamine levels produced during the sleep period.(135) It also plays a vital role in regulating microglial function. Microglia exposure to histamine will lead to proinflammatory conditions.(136)Figure 7 depicts the physiological role of histamine.

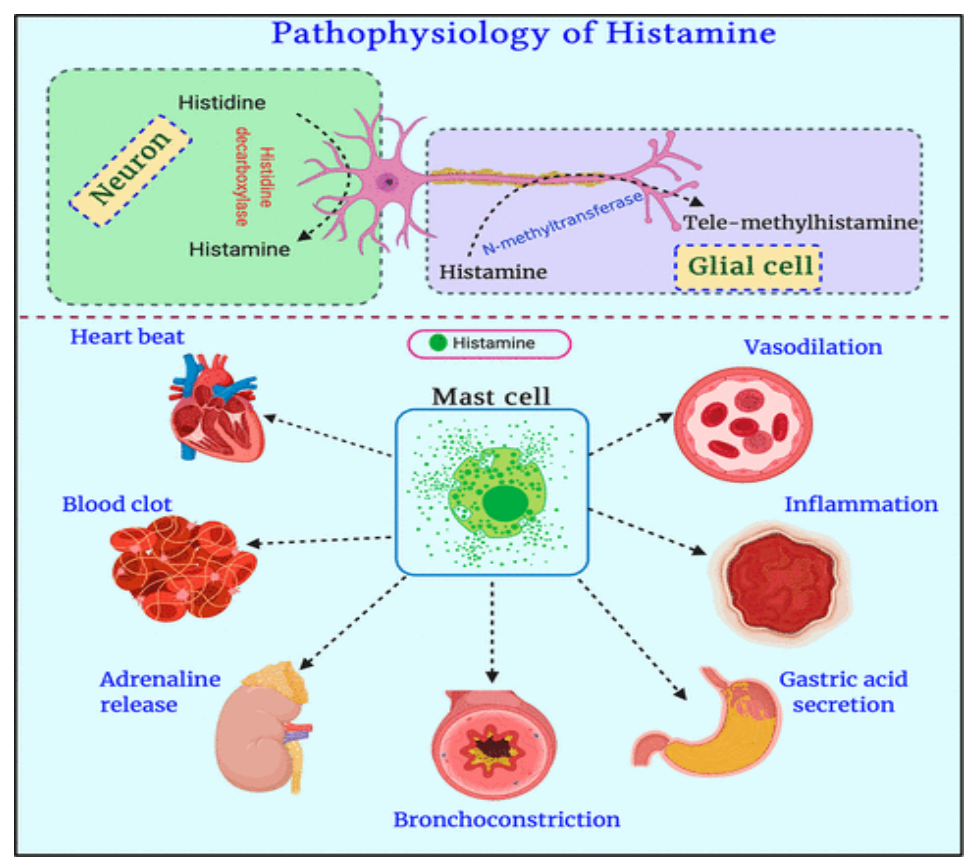

Figure 7. Pathophysiology of histamine in neurons and its role in mast cell (images drawn using Biorender.com).

Despite its importance, histamine has not been given due attention as a biomarker for neurological disorders when compared to the other biogenic amines. This may be because of its moderate action in neurotransmission. Alterations in the release of 
histamine levels lead to several neurological disorders such as schizophrenia, amyotrophic lateral sclerosis, and health for scombroid poisoning characterized by different symptoms such as diarrhea, headache, vomiting, nausea, itching, red rash, etc. Histamine poisoning or scombroid poisoning has been reported by several researchers.(137) A large percentage of the electrochemical sensors developed for the detection of histamine are reported for the analysis of scombroid poisoning. $(137,138,147-151,139-146)$ Several histamine sensors have been reported for detection of fish freshness. However, there are very few reports of electrochemical sensors for histamine detection in the brain as a neurotransmitter molecule or in cells. A carbon fiber microelectrode-based sensor has been successfully employed to detect both histamine and 5-hydroxytryptamine from mast cells. It was demonstrated that at the individual cell level, histamine could be detected with a detection limit as low as $1.4 \mu \mathrm{M} .(152)$ Similarly, a carbon-based electrode was modified using a gel comprising a blend of an osmium compound and poly(vinylpyridine) along with the enzyme mixture of histamine oxidase and horseradish peroxidase. The enzyme-based sensor reported a detection limit of $23 \mathrm{nM}$ and stability over 2 weeks. The sensor was used for real-time determination of histamine released from mast cells.(153) A hybrid nanointerface comprising graphene nanoribbons and silver nanoparticles deposited on pyrolytic graphite electrode was reported to catalyze the oxidation of histamine with high efficiency. The sensor exhibited a detection limit of $49 \mathrm{nM}$ and two linear ranges between 1 and 50 and $60-500 \mu \mathrm{M}$ of histamine. The sensor exhibited good recovery values greater than $99 \%$ from blood plasma samples indicating its promise for clinical applications. A combination of graphene with silver nanoparticles resulted in a very high surface area available for the catalytic activity of this sensing material surface. Hence, the sensor demonstrated an excellent detection limit with good stability for 24 days and 30 scan repetitions.(141) A composite of multiwalled carbon nanotubes and a nickel-benzetricarboxylate metal-organic framework was coated on the surface of a glassy carbon electrode. This sensor detected histamine with a detection limit of 0.41 $\mu \mathrm{M}$ in spiked human urine samples.(154) Carbon paste electrode incorporating molecularly imprinted polymethacrylic acid specific for histamine was used to quantify histamine between 0.1 and $7 \mathrm{nM}$ with a detection limit of $74 \mathrm{pM}$, the lowest value reported so far. The sensor exhibited a high degree of specificity toward histamine in spiked serum samples containing other neurotransmitters such as serotonin and dopamine.(150) Boron-doped diamond electrodes were employed for amperometric recording of signals due to histamine release from ECL cells that was not affected by the presence of other neurotransmitters.(155) However, the potential used for the measurements was $1.3 \mathrm{~V}$, which may cause fouling of the electrode as well as produce signals from other electroactive species that may be present in the sample that increases the risk of erroneous results. Fast scan cyclic voltammetry was employed for determination of histamine release in rat premammillary cells post stimulation of the forebrain bundle.(156) In an interesting strategy, the enzyme methylamine dehydrogenase was engineered to replace phenylalanine residue in the alpha subunit by alanine using site-directed mutagenesis. The engineered enzyme was immobilized on a 
polypyrrole film coated electrode and used for the determination of histamine. Though this sensor did not employ any nanointerface, the engineered enzyme exhibited a 4-fold increase in the sensitivity of the sensor toward histamine, thereby opening up new avenues for the use of engineered biomolecules for improving the histamine sensor performance.(145) It has been recently reported that during the electrochemical detection of histamine generally carried out at potentials exceeding $1 \mathrm{~V}$, a one-electron transfer occurs from the imidazole ring of histamine resulting in the formation of a radical. This radical undergoes subsequent electropolymerization under the influence of the applied potential resulting in electrode fouling. This presents a huge challenge in the determination of histamine. It has been suggested that use of Nafion and similar coatings on the electrode could serve to avoid electrode fouling.(157) The limited literature on histamine determination toward diagnosis of neuronal disorders clearly indicates the existence of an enormous opportunity for the application of electrochemical histamine sensors toward quantification of histamine as a neurotransmitter, especially for real-time monitoring.

\section{Epinephrine (EP)}

This neurotransmitter is also called the "fight or flight hormone" or "adrenaline" and raises the glucose levels.(158) Normal levels of adrenaline in blood are at the nanomolar levels. This molecule has been unfortunately been misused in sports to enhance performance of athletes. As a result, the World Anti-Doping Agency (WADA) has banned the consumption of EP during competitive games. A change in the levels of this neurotransmitter leads to several brain disorders such as schizophrenia, cerebral malaises, and Parkinsonism. It has many uses in disease treatment. It can be used to enhance the heart beat rate and can be employed in the management of bronchitis, bronchial asthma, allergies, and emphysema. It also can be used as blood coagulation promoter and constrictor of blood vessels on skin and mucous membranes for immediate control of bleeding. It decreases the absorption of local anesthetics into the bloodstream leading to reduced toxicity, reduction of surgical blood loss, and prolonged medical action. However, the major setback for the electrochemical detection of epinephrine is that it can undergo oxidation easily, with the occurrence of an irreversible redox reaction as well as interference from ascorbic acid during its detection.(159) To overcome these limitations, Tsele et al. have performed the electrochemical studies using $\mathrm{TiO}_{2^{-}}$and $\mathrm{RuO}_{2}$-doped polyaniline films on multiwalled carbon nanotubes to evaluate EP in pharmaceutical samples.(158) In another study, a detection limit of 30 $\mathrm{nM}$ of epinephrine was reported by employing functionalized multiwalled carbon nanotubes with the biocompatible polymer chitosan that was drop-casted on a glassy carbon electrode for epinephrine analysis using the differential pulse voltammetric technique.(159) Similarly, brilliant cresyl blue electrochemically polymerized on a graphene coated glassy carbon electrode (Figure 8A) was employed for EP detection in pharmaceutical samples.(160) Palladium nanoparticles electrodeposited on a glassy carbon electrode was used to quantify EP in the presence of ascorbic acid.(161) The electrooxidation kinetics of EP was improved using a mercaptopropionic acid/gold 
nanoparticles/cystamine modified gold electrode when compared to the bare gold electrode as well as gold nanoparticles and cystamine modified electrodes. A linear range of $0.1-700 \mu \mathrm{M}$ and $0.1-800 \mu \mathrm{M}$ was reported for the $2 \mathrm{D}$ and $3 \mathrm{D}$ template of the electrode modification respectively(162) in the presence of the potential interferents ascorbic acid and uric acid. Different electrochemical methods, namely, differential pulse voltammetry, linear sweep voltammetry, and chronoamperometry, performed using nanoporous gold thin films prepared by sputter and electrochemical deposition techniques were also employed for EP detection (Figure 8C). Comparing to bulk $\mathrm{Au}$ electrodes, nanoporous Au electrodes demonstrated excellent electrocatalytic behavior toward epinephrine electrooxidation and enabled irreversible oxidation of EP, thus resulting in lower detection limits $(2.43 \mu \mathrm{M})$ and excellent stability over a month.(163)

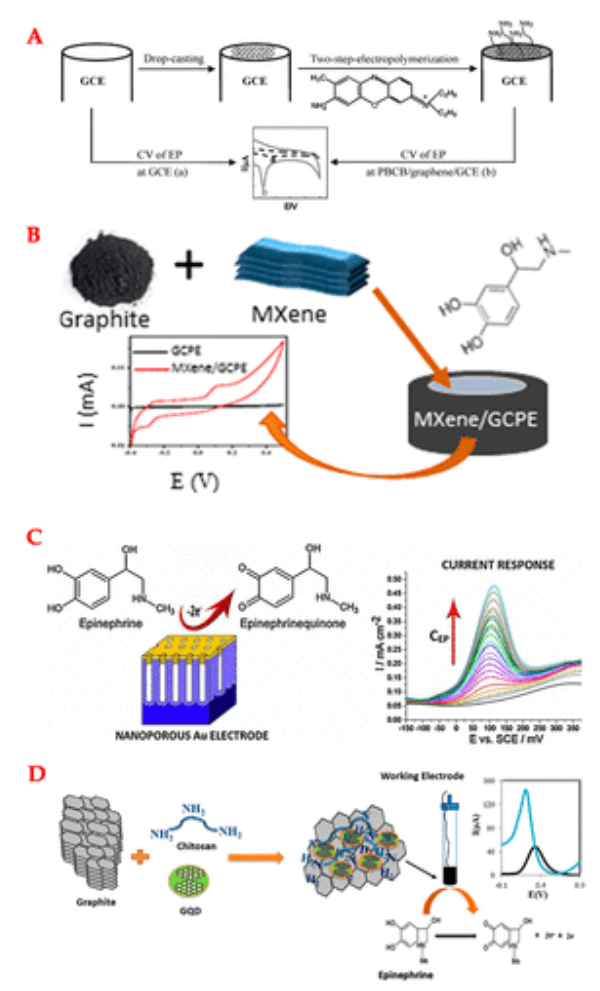

Figure 8. Role of (A) graphene.(160) Reprinted from ref (160). Copyright 2016 with permission from Elsevier. (B) MXene-graphite nanocomposite.(164) Reprinted with permission from ref (164). Copyright 2019 American Chemical Society. (C) Nanoporous gold thin films.(163) Reprinted from ref (163). Copyright 2016 with permission from Elsevier. (D) Graphene quantum dots.(165) Reprinted from ref (165). Copyright 2018 with permission from Elsevier in the electrochemical detection of epinephrine.

A carbon paste electrode modified with multiwalled carbon nanotubes was used to evaluate epinephrine in the presence of ascorbic acid and uric acid.(166) A low detection limit of $0.34 \mathrm{nM}$ was reported by using hybrid nanointerface of graphene oxide consisting of $\mathrm{TiO}_{2}$-Au nanoclusters.(167) Mesoporous carbon was also employed for epinephrine analysis in the presence of uric acid.(168) Graphene oxide-ZnO nanorods were cast on screen-printed electrodes and used for epinephrine detection in 
0.1 M PBS at pH 7.(169) Recently, a MXene-graphite composite paste electrode was reported for epinephrine analysis (Figure $8 \mathrm{~B})$. MXenes are two-dimensional structures of transition metal carbides or nitrides. Two linear ranges between 0.02 and $10 \mu \mathrm{M}$ and $10-100 \mu \mathrm{M}$ were reported for this sensor in $0.2 \mathrm{M}$ phosphate buffer solution of $\mathrm{pH}$ 7.4.(164) Graphene quantum dots and chitosan admixture were drop cast on a carbon paste electrode (Figure 8D) and employed for the analysis of epinephrine with a limit of detection of $0.3 \mathrm{nM} .(165)$ Chemically reduced graphene oxide nanosheets were also reported in recent times for the electrochemical detection of EP in biological serum samples.(170) As EP has been used in medical practice as an emergency medicine, development of robust and selective detection of EP represents a huge prospect in clinical practice.

\section{Norepinephrine (NEP)}

Norepinephrine is one of the most important catecholamine neurotransmitters, which mediates several functions in human body. Because of its action on pupil dilation, heart rate, and blood pressure, WADA has prohibited the usage of the norepinephrine by athletes.(171) NEP plays a vital role in several functions like learning, memory, and the sleep-wake cycle. Norepinephrine activity has been reported in several neurological disorders that includes Alzheimer's disease, Parkinson's disease, hyperactivity, and schizophrenia.(172,173) The major role of NEP is in the conversion of glycogen to glucose in the liver, which helps to increase the energy production by converting fats to fatty acids.(174) The methods so far developed for the detection of the NEP are flow injection analysis,(175,176) high performance liquid chromatography (HPLC),(177) spectrophotometry,(178) chemiluminescence, (179) gas chromatography,(180) and fluorometry.(181) But all these methods are very expensive and time-consuming. These drawbacks can be overcome by the usage of electrochemical biosensors for the selective determination of NEP. Recently, Kunda et al. have reported an enzymeless sensor for the detection of NEP using $\mathrm{MoO}_{3}$ nanowires with a short response time of $2 \mathrm{~s}(\underline{173)}$ (Figure 9A). Molecularly imprinted (MIP) sensors have also attracted considerable interest in the field of electrochemistry. Apart from metal and metal oxides, carbon nanotubes have been used for fast and rapid electron transfer and to achieve excess surface coverage. The combination of MIP and carbon nanotubes was utilized for the NEP sensing by modifying the glassy carbon electrode. This sensor exhibited a good linear range from $99 \mathrm{nM}$ to $15 \mu \mathrm{M}$ with a detection limit of $33 \mathrm{nM} .(182)$ Interference of epinephrine, uric acid, and ascorbic acid plays a major role in the sensitive determination of NEP. Xinying et al. have successfully reported the detection of NEP in the presence of these interferent molecules in NEP injection.(183) A reduced graphene oxide-manganese oxide CRGO$\mathrm{MnO}_{2}$ nanoparticles-modified electrode was used for the simultaneous detection of NEP and acetaminophen at $\mathrm{pH} 7$ in urine and pharmaceutical samples.(184) A screen-printed carbon electrode was modified with $\mathrm{Co}_{3} \mathrm{O}_{4}$ nanoparticles was successfully tested for the detection of NEP in both serum and urine samples.(185) Another research group had a modified glassy carbon electrode with a graphene sheet and gold nanoparticles. This 
electrode was investigated for the analysis of NEP in the presence of high concentrations of ascorbic acid.(186) Carbon paste electrode modified with $\mathrm{ZrO}_{2}$ nanoparticles (ZONMCPE) (187) 2,2'-[1,2 buthanediylbis (nitriloethylidyne)]-bishydroquinone $(\mathrm{BH})$ and a $\mathrm{TiO}_{2}$ nanoparticles modified carbon paste electrode (BHTME)(188) were used for the simultaneous determination of NEP, folic acid, and acetaminophen. A glassy carbon electrode drop-cast with carbon coated nickel magnetic nanoparticles (C-Ni) were employed for NEP determination.(189) Gold nanoparticles electrodeposited on DNA were also explored for the determination of NEP in the presence of high volumes of ascorbic acid, which is a common interferant in biological samples.(190) $x \mathrm{FeMoO}_{4}$ nanorods synthesized using a hydrothermal method were employed for the analysis of NEP in the concentration ranges of 1-200 $\mu \mathrm{M}$ with a rapid response time of $3 \mathrm{~s} .(191)$ In an interesting strategy, hematoxylin was electrochemically deposited on a glassy carbon electrode and employed for the analysis of NEP and acetaminophen with a low detection limit of $0.14 \mu \mathrm{M}(192)$ using differential pulse voltammetry. Similarly, a glassy carbon electrode modified with poly(cresol red) was successfully used for NEP detection at $\mathrm{pH} 3$ with a low detection limit of $0.2 \mu \mathrm{M}(193)$ in the presence of the potential interferent ascorbic acid. Similarly, carbon nanotubes, magnetic cobalt ferrite $\left(\mathrm{FCo}_{98}\right)$ nanoparticles,(194) and graphene(195) were also utilized for NEP quantification. Recent research reveals that there is a link between stress levels, depression, and NEP. It may be possible to detect and treat depression based on NEP levels. With emergence of new roles of NEP in the biological system, refinement of its quantification strategies is required for meeting the clinical demand.

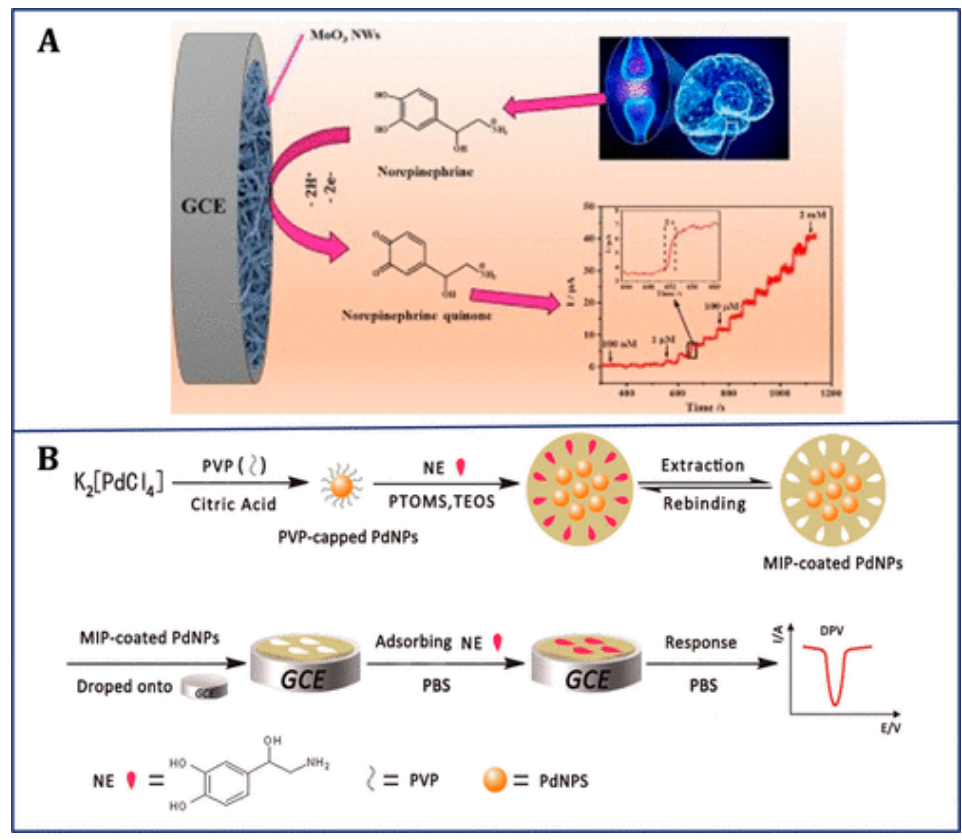

Figure 9. Detection of $\mathrm{NE}$ using(A) $\mathrm{MoO}_{3}$ nanoparticles.(173) Reprinted from ref (173). Copyright 2017 with permission from Elsevier. (B) Palladium nanoparticles.(196) Reprinted from ref (196). Copyright 2015 with permission from Elsevier. 


\section{Gamma Aminobutyric Acid (GABA)}

There are other neurotransmitters such as gamma aminobutryric acid (GABA), an inhibitory neurotransmitter synthesized from glutamate and found in the central nervous system. GABA is known to cause hyperpolarization by causing an efflux of potassium ions or influx of anions into the neuronal cells.(197) It plays a regulatory role in the development, proliferation, migration, and differentiation of neurons as well as influences extension of neuritis. Deregulation of GABA signaling has been associated with neurological disorders such as epilepsy, Parkinson's, and Huntington's diseases. Quantification of GABA through electrochemical methods is a challenge owing to its nonredox active nature. The monomer 5-fluorouracil- $N$-acetylacrylamide and crosslinker ethylene glycol dimethacrylate were used to develop a molecularly imprinted polymer system specific for GABA and deposited over a multiwalled carbon nanotube deposited pencil graphite electrode. The sensor exhibited a linear range between 0.75 and $205 \mathrm{ng} / \mathrm{mL}$ for GABA in human serum and cerebrospinal fluid samples.(198) Recently, a new technique called nano-ITIES (nanointerface between two immiscible electrolyte solutions) was reported for quantification of GABA. The system used a dibenzo-18-crown-6 ionophore, which exhibits a strong affinity for cationic species to generate a current due to potential-driven ion transfer across the pipet-electrolyte interface. GABA possesses no net charge at neutral $\mathrm{pH}$ where it exists as a zwitterion. To transform GABA to a cationic species, octanoic acid was introduced to reduce $\mathrm{pH}$ enabling detection of GABA. The detection range of GABA was $0.25-1$ $\mathrm{mM}$ using this method.(199) There still exist possibilities to fine-tune this strategy to achieve a higher sensitivity and wider detection ranges. In another interesting strategy, an array comprising two sensors: one for glutamate using the enzyme glutamate oxidase as the sensing element (sensor 1) and the other site using the enzyme mix GABase containing $\gamma$-aminobutyric acid aminotransferase (GABGT), succinic semialdehyde dehydrogenase (SSDH) and glutamate oxidase (sensor 2) were used. The end product at both working electrodes was $\mathrm{H}_{2} \mathrm{O}_{2}$, which generated the signal. The signal from sensor 1 was solely due to glutamate present in the sample, while the signal generated at the sensor 2 was due to the combination of glutamate and GABA. Subtraction of the two signals corresponded to the signal proportional to the GABA in the sample.(46) Considering the need for portable point-of-care devices for quantification of GABA, concerted efforts toward harnessing the advantages of nanointerfaces along with smart strategies for electrochemical detection are required.

Table 1 summarizes the different electrochemical strategies that have been reported in the past two decades for the quantification of different neurotransmitters. It is evident that despite the volume of reports, opportunities still exist to develop sensors with improved performance in the context of neurotransmitter analysis. 
Table 1. Electrochemical Strategies Reported in the Past Two Decades for the Quantification of Different Neurotransmittersa

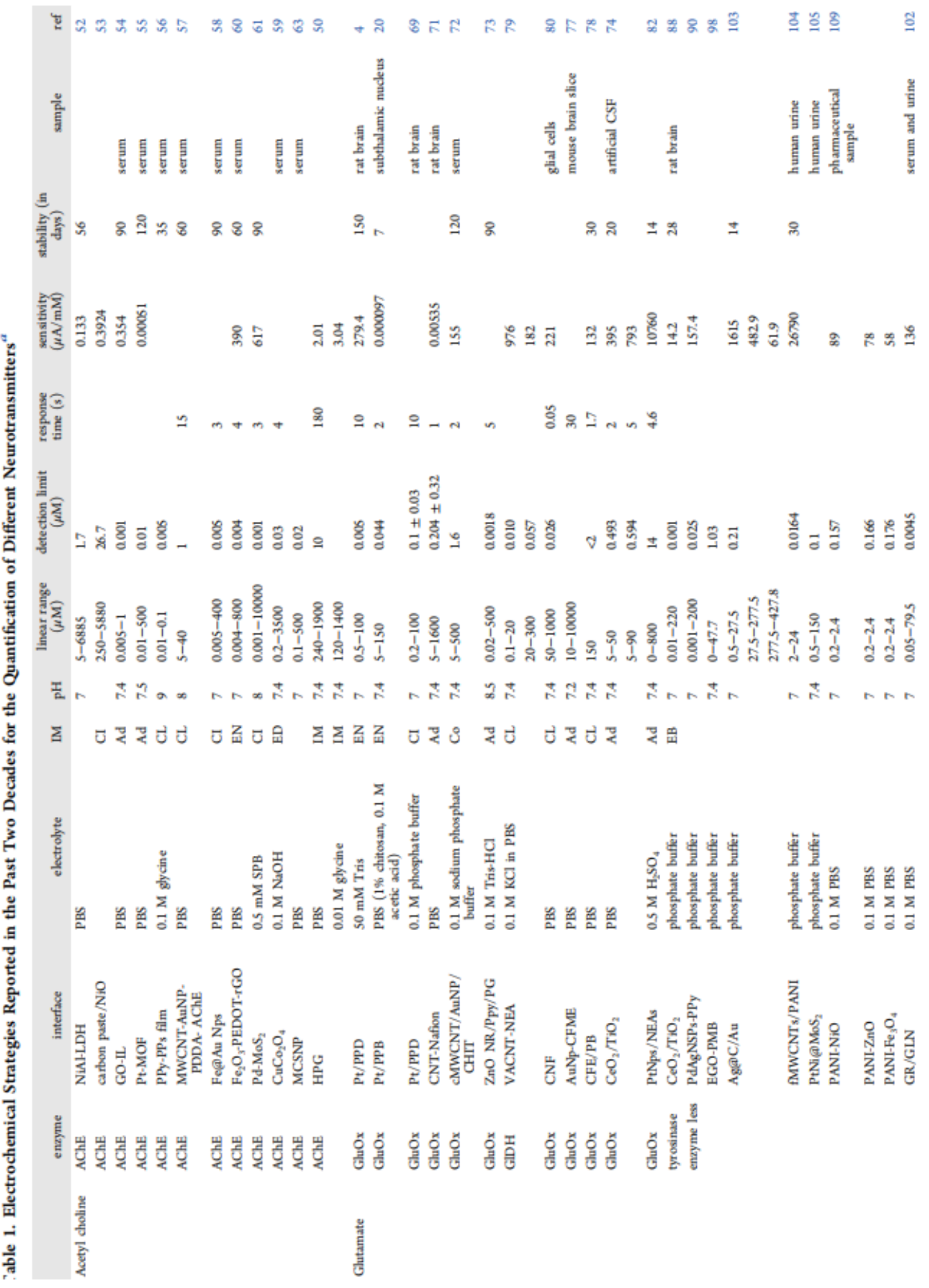


$\mathrm{IM}=$ immobilization, $\mathrm{PPD}$ : poly (phenylenediamine, $\mathrm{PB}=$ Prussian Blue, $\mathrm{CFE}=$ carbon fiber electrode, $\mathrm{AsOx}=$ ascorbate oxide, $\mathrm{GluOx}=$ glutamate oxidase, $\mathrm{GO}-\mathrm{IL}=$ graphene oxide-ionic liquid, $\mathrm{AD}=$ adsorption, $\mathrm{CL}=$ cross-linking, $\mathrm{EN}=$ entrapment, $\mathrm{CI}=$ coimmobilization, $\mathrm{MOF}=$ metal-organic framework, $\mathrm{MWCNT}=$ multiwalled carbon nanotubes, AuNP $=$ gold nanoparticles, PDDA $=$ polydiallyldimethyl ammonium chloride, $\mathrm{HPG}=$ highly porous gold, $\mathrm{SPB}=$ sodium phosphate buffer, MCSNP = magnetic core shell nanoparticles, GIDH = glutamate dehydrogenase, VACNT-NEA = vertically aligned carbon nanotube-nanoelectrode array, $\mathrm{CNF}=$ carbon nanofiber, $\mathrm{CHIT}=$ chitosan, $\mathrm{EB}=$ electrostatic binding, EGO-PMB = electrochemically reduced graphene oxide-poly methylene blue, fMWCNTs $=$ functionalized multiwalled carbon nanotubes, $\mathrm{PANI}=$ polyaniline, $\mathrm{PtNi}=$ platinum-nickel nanoparticles, $\mathrm{MoS}_{2}=$ molybdenum sulfide nano sheets, GR/GLN = graphite-gelatin, $\mathrm{Cu}-\mathrm{TCCP}=\mathrm{Cu}$-tetrakis (4-carboxyphenyl) porphyrin $(\mathrm{TCPP})$, AuNps $=$ gold nanoparticles, $\mathrm{p}(\mathrm{XA})=$ poly (xanthurenic acid), RGO = reduced graphene oxide, $\mathrm{Au} @ \mathrm{Pd}=$ gold-palladium core shell nanoparticles, G- $g$-PLA-pd = graphene oxide-poly lactic acid-palladium nanoparticles, EPPGS = edge plane pyrolytic graphite sensor, fMWCNT = functionalized multi walled carbon nanotubes, $\mathrm{PBCB}=$ poly brilliant cresyl blue, ATO $=$ antimony doped tin oxide, MIP $=$ molecularly imprinted polymers, ZONMCPE $=$ carbon paste electrode modified with $\mathrm{ZrO}_{2}$ nanoparticles, BHTME = buthanediylbis (nitriloethylidyne)]-bis-hydroquinone $(\mathrm{BH})$ and $\mathrm{TiO}_{2}$ nanoparticles modified carbon paste electrode, $\mathrm{C}-\mathrm{Ni}=$ carbon coated nickel magnetic nanoparticles, $\mathrm{CRGO}-\mathrm{MnO}_{2}=$ reduced graphene oxide-manganese oxide, $\mathrm{FCo}_{98}=$ ferrite cobalt nanoparticles, $\mathrm{GS}=$ graphene sheet, GNPS = gold nanoparticles.

\section{Simultaneous Analysis of NTs}

Each of the neurotransmitter discussed above has a different pathophysiological role in each of the brain disorders. In most of the diseases, several NTs play vital roles. So simultaneous measurement of these NTs at the same site is clinically relevant to understand the disease mechanism, pathophysiology, (37) as well as effective diagnosis. Single NT measurement and quantification may not be sufficient for early and accurate diagnosis, and to treat the disease conditions. $(21,200)$ Considerable effects have been made by several research groups for simultaneous analysis of the NTs, which is very challenging when compared to the single NT detection. Differential pulse voltammetry has been the extensively investigated technique for simultaneous analysis of NTs. $(200,201)$ A few research groups have employed square wave voltammetry also.(202) Several combinations of NTs such as dopamine and EP,(25) dopamine and serotonin $(119)$ have been reported using multiwalled carbon nanotubes as the interface material in the presence of the common interferents such as uric acid and ascorbic acid. Using the same interface, dopamine and epinephrine were individually detected in pharmaceutical samples.(203) The same combination of NTs was also measured using silver nanoparticles in serum samples.(200) There are reports of simultaneous measurement of dopamine and serotonin using carbon nanotubes intercalated graphite electrodes,(204) carbon nanotubes,(205) carbon microeletrodes,(206) polypyrrole-gold nanocluster,(207) gold nanoparticles(116) and graphene oxide-porphyrin.(208) The 
combination of EP and NEP have been detected using pyrolytic graphite, $(209)$ iron oxide $(210)$ while reports are available on the simultaneous detection of NEP and serotonin using carbon nanotubes, (211) multiwalled carbon nanotubes, and zinc oxide as a hybrid nanointerface.(202) EP, NEP, and dopamine have been simultaneous determined using an interface of palladium nanoparticles.(212) Other combinations of NTs that have been detected include serotonin and histamine using boron doped diamond electrodes,(213) and $\mathrm{ACh}$ and dopamine using a $\mathrm{Cu} @ \mathrm{Cu}_{2} \mathrm{O}$-mesoporous carbon composite.(214) Gold nanoclusters with a carbon ionic liquid crystal was recently reported for the simultaneous analysis of dopamine, EP, and NEP along with anti-Parkinson's drugs.(215) Many brain disorders exhibit different pathophysiological manifestations, but they also share interconnected signaling pathways with each other based on the levels of the different NT molecules. Even a subtle shift from the tightly regulated NT levels can result in a neuronal disorder. Hence, there is an unmet requirement for the development of efficient methods for the simultaneous analysis of different combinations of NTs.

Direct measurement of NTs in vivo currently remains a challenge for the clinicians. Though evolution of different generations of electrochemical biosensors has resulted in improved detection limits with very quick response times, the size of the electrode probe is a critical factor in in vivo measurements. Fabrication of miniaturized electrodes without compromising the sensing characteristics is a challenge. The movement of the test subject during the measurement can lead to difficulties in estimation of the NT concentrations due to movement-associated artifacts. Another factor influencing in vivo measurements is the variations in NT levels in different extracellular and intracellular fluids, serum, as well as in neurons. Though several attempts toward design of implantable sensors for direct measurement of NTs have been reported by some research groups, the probe size remains a stumbling block. Currently, concerted efforts are underway across the globe to develop ultrasmall probes that can be implantable inside the brain for continuous monitoring of NTs, which can transform the clinical diagnosis scenario in the coming years.

\section{Conclusions and Future Perspectives}

Dysregulation of neurotransmitter levels have been implicated in many neurological diseases. Quantification of neurotransmitter levels, therefore, assumes clinical significance. Though several strategies exist for quantification of neurotransmitters, the need of the hour is to develop sensors with high sensitivity, wide detection range, and rapid response time. This review focuses mainly on the electrochemical detection of neurotransmitters by utilizing nanomaterials as interfaces. The information given in this article is comprehensively arranged after thorough and careful consideration of articles published in peer review journals for the detection of neurotransmitters over the past two decades. Several combinations of nanoparticles have been employed as interface materials to aid rapid electron transfer at the electrode-electrolyte interface as well as to serve as high surface area immobilization matrices for the enzymes. Both enzymatic 
and nonenzymatic approaches have been reported using both in vitro and in vivo experiments. We have focused on the analysis of individual NTs as analyte molecules by using different interface materials. In addition, a short discussion about simultaneous detection of NTs or multiple NT detection strategies is also presented. Even though an enormous number of sensors have been reported for NT analysis employing nanomaterial interfaces, there is a need to design new strategies with hybrid interface materials for rapid, sensitive, and selective determination of NTs in small sample volumes. However, disposable platforms and lab-on-a-chip options that could operate with minimal sample volume are yet to be realized in the context of neurotransmitter detection. Another facet that remains relatively unexplored is the possibility of development of a panel that can probe multiple neurotransmitters simultaneously for effective analysis. Electrochemical biosensors have significant applications due to their low-cost and portability, and they can be integrated into handheld devices, which can be deployed on-site and extended for possible real-time analysis in a forthcoming era. Concerted efforts to harness the potential of nanoparticles with nanofabrication strategies integrated with the electrochemical detection platform can offer the way forward in neurotransmitter-based diagnostics. Stability and reliability still limit the performance of electrochemical sensors for determination of neurotransmitters. Especially in the case of biosensors, the enzyme-immobilized surface tends to degrade over time, which results in a reduction in sensor performance. Systematic studies focused on long-term stability would be useful in providing further required information about implementation of nanomaterials for NT sensors. The electrochemical detection strategies developed so far using nanomaterials clearly have shown beneficial improvements in the sensing characteristics and therefore have immense potential for use in clinics for the diagnosis and monitoring of neurological disorders.

\section{References}

This article references 219 other publications.

1. 1Venton, B. J. and Wightman, R. M. (2003) Psychoanalytical Electrochemistry: Dopamine and Behavior. Anal. Chem. 75 (19), 414A-421A, DOI: 10.1021/ac031421c

2. Shen, M. and Colombo, M. L. (2015) Electrochemical Nanoprobes for the Chemical Detection of Neurotransmitters. Anal. Methods 7(17), 7095-7105, DOI: 10.1039/C5AY00512D

3. 3Shadlaghani, A., Farzaneh, M., Kinser, D., and Reid, R. C. (2019) Direct Electrochemical Detection of Glutamate, Acetylcholine, Choline, and Adenosine Using Non-Enzymatic Electrodes. Sensors 19 (3), 447, DOI: $10.3390 / \mathrm{s} 19030447$ 
4. Tian, F., Gourine, A. V., Huckstepp, R. T. R., and Dale, N. (2009) A Microelectrode Biosensor for Real Time Monitoring of L-Glutamate Release. Anal. Chim. Acta 645 (1-2), 86-91, DOl: 10.1016/j.aca.2009.04.048

5. Moon, J. M., Thapliyal, N., Hussain, K. K., Goyal, R. N., and Shim, Y. B. (2018) Conducting Polymer-Based Electrochemical Biosensors for Neurotransmitters: A Review. Biosens. Bioelectron. 102, 540-552, DOI: 10.1016/j.bios.2017.11.069

6. Obi-Nagata, K. t. and Yusuke Hayashi-Takagi, A. (2019) Synaptic Functions and Their Disruption in Schizophrenia: From Clinical Evidence to Synaptic Optogenetics in an Animal Model. Proc. Japan Acad. Ser.

B 95 (5), 179- 197, DOI: 10.2183/pjab.95.014

7. Xiao, G., Xu, S., Song, Y., Zhang, Y., Li, Z., Gao, F., Xie, J., Sha, L., Xu, Q., Shen, Y., and Cai, X. (2019) In Situ Detection of Neurotransmitters and Epileptiform Electrophysiology Activity in Awake Mice Brains Using a Nanocomposites Modified Microelectrode Array. Sens. Actuators, B 288, 601-610, DOI: 10.1016/j.snb.2019.03.035

8. Cao, Q., Wang, Y., Chen, B., Ma, F., Hao, L., Li, G., Ouyang, C., and Li, L. (2019) Visualization and Identification of Neurotransmitters in Crustacean Brain via Multifaceted Mass Spectrometric Approaches. ACS Chem. Neurosci. 10 (3), 1222-1229, DOI: 10.1021/acschemneuro.8b00730

9. Ayano, G. (2016) Common Neurotransmitters: Criteria for Neurotransmitters, Key Locations, Classifications and Functions. Adv. Psychol. Neurosci. 1 (1), 1- 5, DOI: 10.11648/j.apn.20160101.11

10. Agnihotri, S. K., and Kesari, K. K. (2019) Mechanistic Effect of Heavy Metals. In Neurological Disorder and Brain Cancer BT - Networking of Mutagens in Environmental Toxicology (Kesari, K. K., Ed.) pp 25- 47, Springer International Publishing, Cham. DOI: 10.1007/978-3-31996511-6_2.

11. Lleó, A., Parnetti, L., Belbin, O., and Wiltfang, J. (2019) Has the Time Arrived for Cerebrospinal Fluid Biomarkers in Psychiatric Disorders?. Clin. Chim. Acta 491, 81- 84, DOI: 10.1016/j.cca.2019.01.019

12. Vöö, S., and Bomanji, J. (2019) 18F-FDG PET/CT Indications, Pitfalls and Limitations in Brain Imaging BT. In PET/CT in Brain Disorders (Fraioli, F., Ed.) pp 91- 101, Springer International Publishing, Cham. DOI: 10.1007/9783-030-01523-7_9. 
13. Levigoureux, E., Bouillot, C., Baron, T., Zimmer, L., and Lancelot, S. (2019) PET Imaging of the Influence of Physiological and Pathological aSynuclein on Dopaminergic and Serotonergic Neurotransmission in Mouse Models. CNS Neurosci. Ther. 25 (1), 57-68, DOI: 10.1111/cns.12978

14. Mecca, A. P., Smith, G. S., Gatchel, J. R., and Donovan, J. N. (2019) Advances in the molecular imaging of alzheimer's disease: neurobiological mechanisms of disease and neuropsychiatric symptoms: Session 209. Am. J. Geriatr. Psychiatry 27, S17- S18, DOI: 10.1016/j.jagp.2019.01.158

15. Hermann, W. (2019) Chapter 24 - Cerebral Imaging in Wilson Disease. In Clinical and Translational Perspectives on Wilson Disease (Kerkar, N., Roberts, E. A. B. T.-C., and T. P, W. D., Eds.) pp 271- 278, Academic Press. DOI: 10.1016/B978-0-12-810532-0.00024-0 .

16. Windels, F., Bruet, N., Poupard, A., Urbain, N., Chouvet, G., Feuerstein, C., and Savasta, M. (2000) Effects of High Frequency Stimulation of Subthalamic Nucleus on Extracellular Glutamate and GABA in Substantia Nigra and Globus Pallidus in the Normal Rat. Eur. J.

Neurosci. 12 (11), 4141-4146, DOI: 10.1046/j.1460-9568.2000.00296.

17. Hascup, E. R., Af Bjerkén, S., Hascup, K. N., Pomerleau, F., Huettl, P., Strömberg, I., and Gerhardt, G. A. (2009) Histological Studies of the Effects of Chronic Implantation of Ceramic-Based Microelectrode Arrays and Microdialysis Probes in Rat Prefrontal Cortex. Brain Res. 1291, 12- 20, DOI: 10.1016/j.brainres.2009.06.084

18. Kennedy, R. T., Watson, C. J., Haskins, W. E., Powell, D. H., and Strecker, R. E. (2002) In Vivo Neurochemical Monitoring by Microdialysis and Capillary Separations. Curr. Opin. Chem. Biol. 6 (5), 659- 665, DOI: 10.1016/S1367-5931(02)00373-3

19. Hascup, E. R. and Hascup, K. N. (2014) Electrochemical Techniques for Subsecond Neurotransmitter Detection in Live Rodents. Comp. Med. 64 (4), 249- 255

20. Ganesana, M., Trikantzopoulos, E., Maniar, Y., Lee, S. T., and Venton, B. J. (2019) Development of a Novel Micro Biosensor for in Vivo Monitoring of Glutamate Release in the Brain. Biosens. Bioelectron. 130, 103-109, DOI: 10.1016/j.bios.2019.01.049

21. Si, B. and Song, E. (2018) Recent Advances in the Detection of Neurotransmitters. Chemosensors 6 (1), 1, DOI: 10.3390/chemosensors6010001 
22. Castaño-Álvarez, M., Fernández-Abedul, M. T., Costa-García, A., Agirregabiria, M., Fernández, L. J., Ruano-López, J. M., and BarredoPresa, B. (2009) Fabrication of SU-8 Based Microchip Electrophoresis with Integrated Electrochemical Detection for Neurotransmitters. Talanta 80 (1), 24-30, DOI: 10.1016/j.talanta.2009.05.049

23. Ling, X., Shi, R., Zhang, J., Liu, D., Weng, M., Zhang, C., Lu, M., Xie, X., Huang, L., and Huang, W. (2018) Dual-Signal Luminescent Detection of Dopamine by a Single Type of Lanthanide-Doped Nanoparticles. ACS Sensors 3, 1683-1689, DOI: 10.1021/acssensors.8b00368

24. Dutta, S., Ray, C., Mallick, S., Sarkar, S., Sahoo, R., Negishi, Y., and Pal, T. (2015) A Gel-Based Approach to Design Hierarchical CuS Decorated Reduced Graphene Oxide Nanosheets for Enhanced Peroxidaselike Activity Leading to Colorimetric Detection of Dopamine. J. Phys. Chem. C 119 (41), 23790-23800, DOI: 10.1021/acs.jpcc.5b08421

25. Aashish, A., Sadanandhan, N. K., Ganesan, K. P., Saraswathy Hareesh, U. N., Muthusamy, S., and Devaki, S. J. (2018) Flexible Electrochemical Transducer Platform for Neurotransmitters. ACS Omega 3 (3), 3489- 3500, DOI: 10.1021/acsomega.7b02055

26. Wei, D., Bailey, M. J. A., Andrew, P., and Ryhänen, T. (2009) Electrochemical Biosensors at the Nanoscale. Lab Chip 9 (15), 2123-2131, DOI: 10.1039/b903118a

27. Zestos, A. G. (2018) Carbon Nanoelectrodes for the Electrochemical Detection of Neurotransmitters. Int. J. Electrochem. 2018, 1- 19, DOI: $10.1155 / 2018 / 3679627$

28. Madhurantakam, S., Jayanth Babu, K., Balaguru Rayappan, J. B., and Krishnan, U. M. (2017) Fabrication of Mediator-Free Hybrid NanoInterfaced Electrochemical Biosensor for Monitoring Cancer Cell Proliferation. Biosens. Bioelectron. 87, 832- 841, DOI: 10.1016/j.bios.2016.09.039

29. Pundir, S., Chauhan, N., Narang, J., and Pundir, C. S. (2012) Amperometric Choline Biosensor Based on Multiwalled Carbon Nanotubes/Zirconium Oxide Nanoparticles Electrodeposited on Glassy Carbon Electrode. Anal. Biochem. 427 (1), 26-32, DOI: 10.1016/j.ab.2012.04.027

30. Jayanth Babu, K., Sasya, M., Nesakumar, N., Shankar, P., Gumpu, M. B., Ramachandra, B. L., Kulandaisamy, A. J., and Rayappan, J. B.

B. (2017) Non-Enzymatic Detection of Glucose in Fruits Using TiO2?Mn3O4 
Hybrid Nano Interface. Appl. Nanosci. 7, 309- 316, DOI: 10.1007/s13204017-0571-1

31. von Gersdorff, H. (2008) Synaptic Transmission. In Neuroscience in Medicine, 3rd ed., pp 95-109, Springer. DOI: 10.1007/978-1-60327-455-5_5 .

32. Fitzsimonds, R. M. and Poo, M. M. (1998) Retrograde Signaling in the Development and Modification of Synapses. Physiol.

Rev. 78 (1), 143-170, DOI: 10.1152/physrev.1998.78.1.143

33. Regehr, W. G., Carey, M. R., and Best, A. R. (2009) Activity-dependent regulation of synapses by retrograde

messengers. Neuron 63 (2), 154-170, DOI: 10.1016/j.neuron.2009.06.021

34. Westfall, T. C. (1977) Local Regulation of Adrenergic Neurotransmission. Physiol. Rev. 57 (4), 659- 728, DOI:

10.1152/physrev.1977.57.4.659

35. Chanaday, N. L. and Kavalali, E. T. (2018) Presynaptic Origins of Distinct Modes of Neurotransmitter Release. Curr. Opin.

Neurobiol. 51, 119-126, DOI: 10.1016/j.conb.2018.03.005

36. Neher, E. and Brose, N. (2018) Dynamically Primed Synaptic Vesicle States: Key to Understand Synaptic Short-Term

Plasticity. Neuron 100 (6), 1283-1291, DOI: 10.1016/j.neuron.2018.11.024

37. Matys, J., Gieroba, B., and Jóźwiak, K. (2020) Recent Developments of Bioanalytical Methods in Determination of Neurotransmitters in Vivo. J. Pharm. Biomed. Anal. 180, 113079, DOI: 10.1016/j.jpba.2019.113079

38. Tavakolian-Ardakani, Z., Hosu, O., Cristea, C., Mazloum-Ardakani, M., and Marrazza, G. (2019) Latest Trends in Electrochemical Sensors for Neurotransmitters: A Review. Sensors 19, 2037, DOI: 10.3390/s19092037

39. Wang, W., Deng, Y., He, X., Xu, L., and Li, Z. (2012) Progress in Preparation and Application of Nanostructured Neurotransmitters Microsensors/Microelectrodes. J. Nanosci.

Nanotechnol. 12 (9), 7301-7306, DOI: 10.1166/jnn.2012.6154

40. Arumugasamy, S. K., Chellasamy, G., Gopi, S., Govindaraju, S., and Yun, K. (2020) Current Advances in the Detection of Neurotransmitters by Nanomaterials: An Update. TrAC, Trends Anal. Chem. 123, 115766, DOI: 10.1016/j.trac.2019.115766

41. Azzouz, A., Goud, K. Y., Raza, N., Ballesteros, E., Lee, S. E., Hong, J., Deep, A., and Kim, K. H. (2019) Nanomaterial-Based Electrochemical 
Sensors for the Detection of Neurochemicals in Biological Matrices. TrAC,

Trends Anal. Chem. 110, 15- 34, DOI: 10.1016/j.trac.2018.08.002

42. Maduraiveeran, G., Sasidharan, M., and Ganesan,

V. (2018) Electrochemical Sensor and Biosensor Platforms Based on

Advanced Nanomaterials for Biological and Biomedical Applications. Biosens.

Bioelectron. 103, 113-129, DOI: 10.1016/j.bios.2017.12.031

43. Durairaj, S., Sidhureddy, B., Cirone, J., and Chen,

A. (2018) Nanomaterials-Based Electrochemical Sensors for in Vitro and in Vivo Analyses of Neurotransmitters. Appl. Sci. 8, 1504, DOI:

10.3390/app8091504

44. Suhito, I. R., Angeline, N., and Kim, T. H. (2019) Nanomaterial-Modified Hybrid Platforms for Precise Electrochemical Detection of Dopamine. BioChip J. 13 (1), 20-29, DOI: 10.1007/s13206-019-3106-x

45. Sanghavi, B. J., Wolfbeis, O. S., Hirsch, T., and Swami, N.

S. (2015) Nanomaterial-Based Electrochemical Sensing of Neurological Drugs and Neurotransmitters. Microchim. Acta 182 (1-2), 1-41, DOI:

10.1007/s00604-014-1308-4

46. Ou, Y., Buchanan, A. M., Witt, C. E., and Hashemi, P. (2019) Frontiers in Electrochemical Sensors for Neurotransmitter Detection: Towards Measuring Neurotransmitters as Chemical Diagnostics for Brain Disorders. Anal. Methods 11, 2738-2755, DOI: 10.1039/C9AY00055K

47. Ou, Y., Buchanan, A. M., Witt, C. E., and Hashemi, P. (2019) Frontiers in Electrochemical Sensors for Neurotransmitter Detection: Towards Measuring Neurotransmitters as Chemical Diagnostics for Brain Disorders. Anal. Methods 11 (21), 2738-2755, DOI: 10.1039/C9AY00055K

48. Xu, C., Wu, F., Yu, P., and Mao, L. In Vivo Electrochemical Sensors for Neurochemicals: Recent Update. ACS Sensors 2019. DOI:

10.1021/acssensors.9b01713 .

49. Carlo, B. (2020) The Covid-19 Virus Double Pathogenic Mechanism. A New Perspective. PrePrints (April), 1-25, DOI:

10.20944/preprints202004.0165.v2

50. Moreira, F. T. C., Sale, M. G. F., and Di Lorenzo, M. (2017) Towards Timely Alzheimer Diagnosis: A Self-Powered Amperometric Biosensor for the Neurotransmitter Acetylcholine. Biosens. Bioelectron. 87, 607-614, DOI: 10.1016/j.bios.2016.08.104 
51. Watanabe, M., Kimura, A., Akasaka, K., and Hayashi, S. (1986) Determination of Acetylcholine in Human Blood. Biochem. Med. Metab. Biol. 36 (3), 355-362, DOI: 10.1016/0885-4505(86)90147-7

52. Wang, L., Chen, X., Liu, C., and Yang, W. (2016) Non-Enzymatic Acetylcholine Electrochemical Biosensor Based on Flower-like NiAl Layered Double Hydroxides Decorated with Carbon Dots. Sens. Actuators, B 233, 199-205, DOI: 10.1016/j.snb.2016.04.062

53. Sattarahmady, N., Heli, H., and Vais, R. D. (2013) An Electrochemical Acetylcholine Sensor Based on Lichen-like Nickel Oxide Nanostructure. Biosens. Bioelectron. 48, 197-202, DOI: 10.1016/j.bios.2013.04.001

54. Albishri, H. M. and Abd El-Hady, D. (2019) Hyphenation of Enzyme/Graphene Oxide-Ionic Liquid/Glassy Carbon Biosensors with Anodic Differential Pulse Stripping Voltammetry for Reliable Determination of Choline and Acetylcholine in Human Serum. Talanta 200 (March), 107-114, DOI: 10.1016/j.talanta.2019.03.028

55. Chauhan, N., Tiwari, S., Narayan, T., and Jain, U. (2019) Bienzymatic Assembly Formed @ Pt Nano Sensing Framework Detecting Acetylcholine in Aqueous Phase. Appl. Surf. Sci. 474, 154-160, DOI: 10.1016/j.apsusc.2018.04.056

56. Aynaci, E., Yasar, A., and Arslan, F. (2014) An Amperometric Biosensor for Acetylcholine Determination Prepared from Acetylcholinesterase-Choline Oxidase Immobilized in Polypyrrole- Polyvinylsulpfonate Film. Sens. Actuators, B 202, 1028-1036, DOI: 10.1016/j.snb.2014.06.049

57. Hou, S., Ou, Z., Chen, Q., and Wu, B. (2012) Amperometric Acetylcholine Biosensor Based on Self-Assembly of Gold Nanoparticles and Acetylcholinesterase on the Sol-Gel/Multi-Walled Carbon Nanotubes/Choline Oxidase Composite-Modified Platinum Electrode. Biosens.

Bioelectron. 33 (1), 44- 49, DOI: 10.1016/j.bios.2011.12.014

58. Chauhan, N. and Pundir, C. S. (2014) Amperometric Determination of Acetylcholine-A Neurotransmitter, by Chitosan/Gold-Coated Ferric Oxide Nanoparticles Modified Gold Electrode. Biosens. Bioelectron. 61, 1- 8, DOI: 10.1016/j.bios.2014.04.048

59. Balasubramanian, P., Balamurugan, T. S. T., Chen, S., and Chen, T. (2019) Facile Synthesis of Spinel-Type Copper Cobaltite Nanoplates for Enhanced Electrocatalytic Detection of Acetylcholine. ACS Sustainable Chem. Eng. 7, 7642- 7651, DOI: 10.1021/acssuschemeng.8b06021 
60. Chauhan, N., Chawla, S., Pundir, C. S., and Jain, U. (2017) An Electrochemical Sensor for Detection of Neurotransmitter-Acetylcholine Using Metal Nanoparticles, 2D Material and Conducting Polymer Modified Electrode. Biosens. Bioelectron. 89, 377-383, DOI: 10.1016/j.bios.2016.06.047

61. Jain, U., Khanuja, M., Gupta, S., Harikumar, A., and Chauhan, N. (2019) Pd Nanoparticles and Molybdenum Disulfide (MoS 2) Integrated Sensing Platform for the Detection of Neuromodulator. Process Biochem. 81, 48- 56, DOI: 10.1016/j.procbio.2019.03.019

62. Ashmawy, N. H., Almehizia, A. A., Youssef, T. A., Amr, A. E. G. E., AlOmar, M. A., and Kamel, A. H. (2019) Novel Carbon/PEDOT/PSS-Based Screen-Printed Biosensors for Acetylcholine Neurotransmitter and Acetylcholinesterase Detection in Human Serum. Molecules 24, 1539, DOI: $10.3390 /$ molecules24081539

63. Mohammadi, S. Z., Beitollahi, H., and Tajik, S. (2018) Nonenzymatic Coated Screen - Printed Electrode for Electrochemical Determination of Acetylcholine. Micro Nano Syst. Lett. 6, 9, DOI: 10.1186/s40486-018-0070-5

64. Chauhan, N., Balayan, S., and Jain, U. (2020) Sensitive Biosensing of Neurotransmitter: 2D Material Wrapped Nanotubes and MnO2 Composites for the Detection of Acetylcholine. Synth. Met. 263, 116354, DOI: 10.1016/j.synthmet.2020.116354

65. Vasylieva, N., Maucler, C., Meiller, A., Viscogliosi, H., Lieutaud, T., Barbier, D., and Marinesco, S. (2013) Immobilization Method to Preserve Enzyme Specificity in Biosensors: Consequences for Brain Glutamate Detection. Anal. Chem. 85 (4), 2507-2515, DOI: 10.1021/ac3035794

66. Okon, S. L. and Ronkainen, N. J. (2017) Enzyme-Based

Electrochemical Glutamate Biosensors. Intech i, 13, DOI: 10.5772/68025

67. Jeffries, C., Pasco, N., Baronian, K., and Gorton, L. (1997) Evaluation of a Thermophile Enzyme for a Carbon Paste Amperometric Biosensor: LGlutamate Dehydrogenase. Biosens. Bioelectron. 12, 225- 232, DOI: 10.1016/S0956-5663(97)85340-7

68. Niswender, C. M. and Conn, P. J. (2010) Metabotropic Glutamate Receptors: Physiology, Pharmacology, and Disease. Annu. Rev. Pharmacol. Toxicol. 50 (1), 295-322, DOI: 10.1146/annurev.pharmtox.011008.145533

69. Rahman, M. A., Kwon, N. H., Won, M. S., Choe, E. S., and Shim, Y. B. (2005) Functionalized Conducting Polymer as an Enzyme-Immobilizing 
Substrate: An Amperometric Glutamate Microbiosensor for in Vivo Measurements. Anal. Chem. 77, 4854-4860, DOI: 10.1021/ac050558v

70. Huang, X. J., Im, H. S., Lee, D. H., Kim, H. S., and Choi, Y. K. (2007) Ferrocene Functionalized Single-Walled Carbon Nanotube Bundles. Hybrid Interdigitated Construction Film for L-Glutamate Detection. J. Phys. Chem. C 111 (3), 1200-1206, DOI: 10.1021/jp065747b

71. Ferreira, N. R., Ledo, A., Laranjinha, J., Gerhardt, G. A., and Barbosa, R. M. (2018) Simultaneous Measurements of Ascorbate and Glutamate in Vivo in the Rat Brain Using Carbon Fiber Nanocomposite Sensors and Microbiosensor Arrays. Bioelectrochemistry 121, 142-150, DOI: 10.1016/j.bioelechem.2018.01.009

72. Batra, B. and Pundir, C. S. (2013) An Amperometric Glutamate Biosensor Based on Immobilization of Glutamate Oxidase onto Carboxylated Multiwalled Carbon Nanotubes/Gold Nanoparticles/Chitosan Composite Film Modified Au Electrode. Biosens. Bioelectron. 47, 496- 501, DOI: 10.1016/j.bios.2013.03.063

73. Batra, B., Yadav, M., and Pundir, C. S. (2016) L-Glutamate Biosensor Based on I-Glutamate Oxidase Immobilized onto ZnO Nanorods/Polypyrrole Modified Pencil Graphite Electrode. Biochem. Eng. J. 105, 428- 436, DOI: 10.1016/j.bej.2015.10.012

74. Özel, R. E., Ispas, C., Ganesana, M., Leiter, J. C., and Andreescu, S. (2014) Glutamate Oxidase Biosensor Based on Mixed Ceria and Titania Nanoparticles for the Detection of Glutamate in Hypoxic Environments. Biosens. Bioelectron. 52, 397-402, DOI: 10.1016/j.bios.2013.08.054

75. Lin, X., Wang, Q., Zhu, S., Xu, J., Xia, Q., and Fu, Y. (2016) A Highly Sensitive Glutamic Acid Biosensor Based on the Determination of NADH Enzymically Generated by L-Glutamic Dehydrogenase. RSC Adv. 6 (51), 45829- 45834, DOI: 10.1039/C6RA07235F

76. Marquitan, M., Mark, M. D., Ernst, A., Muhs, A., Herlitze, S., Ruff, A., and Schuhmann, W. (2020) Glutamate Detection at the Cellular Level by Means of Polymer/Enzyme Multilayer Modified Carbon Nanoelectrodes. J. Mater. Chem. B 8, 3631-3639, DOI: 10.1039/C9TB02461A

77. Wang, Y., Mishra, D., Bergman, J., Keighron, J. D., Skibicka, K. P., and Cans, A. S. (2019) Ultrafast Glutamate Biosensor Recordings in Brain Slices Reveal Complex Single Exocytosis Transients. ACS Chem. Neurosci. 10 (3), 1744-1752, DOI: 10.1021/acschemneuro.8b00624 
78. Salazar, P., Martín, M., O’Neill, R. D., and González-Mora, J.

L. (2016) Glutamate Microbiosensors Based on Prussian Blue Modified

Carbon Fiber Electrodes for Neuroscience Applications: In-Vitro

Characterization. Sens. Actuators, B 235, 117- 125, DOI:

10.1016/j.snb.2016.05.057

79. Gholizadeh, A., Shahrokhian, S., Iraji Zad, A., Mohajerzadeh, S., Vosoughi, M., Darbari, S., Koohsorkhi, J., and Mehran,

M. (2012) Fabrication of Sensitive Glutamate Biosensor Based on Vertically

Aligned CNT Nanoelectrode Array and Investigating the Effect of CNTs

Density on the Electrode Performance. Anal.

Chem. 84 (14), 5932- 5938, DOI: 10.1021/ac300463x

80. Isoaho, N., Peltola, E., Sainio, S., Wester, N., Protopopova, V., Wilson, B. P., Koskinen, J., and Laurila, T. (2017) Carbon Nanostructure Based Platform for Enzymatic Glutamate Biosensors. J. Phys. Chem.

C 121 (8), 4618-4626, DOI: 10.1021/acs.jpcc.6b10612

81. Borisova, T., Kucherenko, D., Soldatkin, O., Kucherenko, I., Pastukhov, A., Nazarova, A., Galkin, M., Borysov, A., Krisanova, N., Soldatkin, A., and El skaya, A. (2018) An Amperometric Glutamate Biosensor for Monitoring Glutamate Release from Brain Nerve Terminals and in Blood Plasma. Anal. Chim. Acta 1022, 113-123, DOI: 10.1016/j.aca.2018.03.015

82. Jamal, M., Xu, J., and Razeeb, K. M. (2010) Disposable Biosensor Based on Immobilisation of Glutamate Oxidase on Pt Nanoparticles Modified Au Nanowire Array Electrode. Biosens. Bioelectron. 26 (4), 1420- 1424, DOI: 10.1016/j.bios.2010.07.071

83. Stephens, M. L., Pomerleau, F., Huettl, P., Gerhardt, G. A., and Zhang, Z. (2010) Real-Time Glutamate Measurements in the Putamen of Awake Rhesus Monkeys Using an Enzyme-Based Human Microelectrode Array Prototype. J. Neurosci. Methods 185 (2), 264- 272, DOI:

10.1016/j.jneumeth.2009.10.008

84. Chen, J. L., Yan, X. P., Meng, K., and Wang, S. F. (2011) Graphene Oxide Based Photoinduced Charge Transfer Label-Free near-Infrared Fluorescent Biosensor for Dopamine. Anal. Chem. 83 (22), 8787-8793, DOI: $10.1021 /$ ac2023537

85. Álvarez-Martos, I. and Ferapontova, E. E. (2016) Electrochemical Label-Free Aptasensor for Specific Analysis of Dopamine in Serum in the Presence of Structurally Related Neurotransmitters. Anal.

Chem. 88 (7), 3608-3616, DOI: 10.1021/acs.analchem.5b04207 
86. Farjami, E., Campos, R., Nielsen, J. S., Gothelf, K. V., Kjems, J., and Ferapontova, E. E. (2013) RNA Aptamer-Based Electrochemical Biosensor for Selective and Label-Free Analysis of Dopamine. Anal. Chem. 85 (1), 121-128, DOI: 10.1021/ac302134s

87. Lin, Y. K. and Yeh, Y. C. (2017) Dual-Signal Microbial Biosensor for the Detection of Dopamine without Inference from Other Catecholamine Neurotransmitters. Anal. Chem. 89 (21), 11178-11182, DOI: 10.1021/acs.analchem.7b02498

88. Njagi, J., Chernov, M. M., Leiter, J. C., and Andreescu, S. (2010) Amperometric Detection of Dopamine in Vivo with an Enzyme Based Carbon Fiber Microbiosensor. Anal. Chem. 82 (3), 989-996, DOI: 10.1021/ac9022605

89. Yang, C., Trikantzopoulos, E., Nguyen, M. D., Jacobs, C. B., Wang, Y., Mahjouri-Samani, M., Ivanov, I. N., and Venton, B. J. (2016) Laser Treated Carbon Nanotube Yarn Microelectrodes for Rapid and Sensitive Detection of Dopamine in Vivo. ACS Sensors 1 (5), 508- 515, DOI: 10.1021/acssensors.6b00021

90. Mahmoudian, M. R., Basirun, W. J., and Binti Alias, Y. (2016) Sensitive Dopamine Biosensor Based on Polypyrrole-Coated Palladium Silver Nanospherical Composites. Ind. Eng. Chem. Res. 55 (25), 6943-6951, DOI: 10.1021/acs.iecr.6b00570

91. Siddiqui, S., Shawuti, S., Sirajuddin, Niazi, J. H., and Qureshi, A. (2019) L -Cysteine-Mediated Self-Assembled Ag-Au Nanoparticles As Fractal Patterns with Bowling-Alley-like Hollow Arrays for Electrochemical Sensing of Dopamine. Ind. Eng. Chem. Res. 58 (19), 8035- 8043, DOI: 10.1021/acs.iecr.9b00016

92. Vellaichamy, B., Periakaruppan, P., and Paulmony, T. (2017) Evaluation of a New Biosensor Based on in Situ Synthesized PPyAg-PVP Nanohybrid for Selective Detection of Dopamine. J. Phys. Chem. B 121 (5), 1118-1127, DOI: 10.1021/acs.jpcb.6b11225

93. Qing, X., Wang, Y., Zhang, Y., Ding, X., Zhong, W., Wang, D., Wang, W., Liu, Q., Liu, K., Li, M., and Lu, Z. (2019) Wearable Fiber-Based Organic Electrochemical Transistors as a Platform for Highly Sensitive Dopamine Monitoring. ACS Appl. Mater. Interfaces 11 (14), 13105-13113, DOI: 10.1021/acsami.9b00115

94. Su, C. H., Sun, C. L., and Liao, Y. C. (2017) Printed Combinatorial Sensors for Simultaneous Detection of Ascorbic Acid, Uric Acid, Dopamine, 
and Nitrite. ACS Omega 2 (8), 4245-4252, DOI:

10.1021/acsomega.7b00681

95. Sun, C. L., Chang, C. T., Lee, H. H., Zhou, J., Wang, J., Sham, T. K., and Pong, W. F. (2011) Microwave-Assisted Synthesis of a Core-Shell MWCNT/GONR Heterostructure for the Electrochemical Detection of Ascorbic Acid, Dopamine, and Uric Acid. ACS Nano 5 (10), 7788-7795, DOI: $10.1021 / \mathrm{nn} 2015908$

96. Fabregat, G., Estrany, F., Casas, M. T., Alemán, C., and Armelin, E. (2014) Detection of Dopamine Using Chemically Synthesized Multilayered Hollow Microspheres. J. Phys. Chem. B 118 (17), 4702- 4709, DOI: 10.1021/jp500959j

97. Zhao, J., Zhang, W., Sherrell, P., Razal, J. M., Huang, X. F., Minett, A. I., and Chen, J. (2012) Carbon Nanotube Nanoweb-Bioelectrode for Highly Selective Dopamine Sensing. ACS Appl. Mater. Interfaces 4 (1), 44- 48, DOI: $10.1021 / \mathrm{am} 201508 \mathrm{~d}$

98. Gorle, D. B. and Kulandainathan, M. A. (2016) Electrochemical Sensing of Dopamine at the Surface of a Dopamine Grafted Graphene Oxide/Poly(Methylene Blue) Composite Modified Electrode. RSC Adv. 6 (24), 19982-19991, DOI: 10.1039/C5RA25541D

99. Kim, D. S., Kang, E. S., Baek, S., Choo, S. S., Chung, Y. H., Lee, D., Min, J., and Kim, T. H. (2018) Electrochemical Detection of Dopamine Using Periodic Cylindrical Gold Nanoelectrode Arrays. Sci.

Rep. 8 (1), 1- 10, DOI: 10.1038/s41598-018-32477-0

100. Jiang, J. and Du, X. (2014) Sensitive Electrochemical Sensors for Simultaneous Determination of Ascorbic Acid, Dopamine, and Uric Acid Based on Au@Pd-Reduced Graphene Oxide Nanocomposites. Nanoscale 6 (19), 11303-11309, DOI: 10.1039/C4NR01774A

101. Qiu, Z., Yang, T., Gao, R., Jie, G., and Hou, W. (2019) An Electrochemical Ratiometric Sensor Based on 2D MOF Nanosheet/Au/Polyxanthurenic Acid Composite for Detection of Dopamine. J. Electroanal. Chem. 835, 123-129, DOI: 10.1016/j.jelechem.2019.01.040

102. Rajkumar, C., Thirumalraj, B., Chen, S. M., and Chen, H. A. (2017) A Simple Preparation of Graphite/Gelatin Composite for Electrochemical Detection of Dopamine. J. Colloid Interface Sci. 487, 149-155, DOI: 10.1016/j.jcis.2016.10.024 
103. Liu, X., Fu, Y., Sheng, Q., and Zheng, J. (2019) Au Nanoparticles Attached Ag@C Core-Shell Nanocomposites for Highly Selective

Electrochemical Detection of Dopamine. Microchem. J. 146, 509- 516, DOI: 10.1016/j.microc.2019.01.023

104. Chang, Y. H., Woi, P. M., and Alias, Y. (2019) The Selective Electrochemical Detection of Dopamine in the Presence of Ascorbic Acid and Uric Acid Using Electro-Polymerised- $\beta$-Cyclodextrin Incorporated fMWCNTs/Polyaniline Modified Glassy Carbon Electrode. Microchem. J. 148, 322-330, DOI: 10.1016/j.microc.2019.04.081

105. Ma, L., Zhang, Q., Wu, C., Zhang, Y., and Zeng, L. (2019) PtNi Bimetallic Nanoparticles Loaded MoS 2 Nanosheets: Preparation and Electrochemical Sensing Application for the Detection of Dopamine and Uric Acid. Anal. Chim. Acta 1055, 17-25, DOI: 10.1016/j.aca.2018.12.025

106. Anithaa, A. C., Lavanya, N., Asokan, K., and Sekar, C. (2015) WO3 Nanoparticles Based Direct Electrochemical Dopamine Sensor in the Presence of Ascorbic Acid. Electrochim. Acta 167, 294-302, DOI: 10.1016/j.electacta.2015.03.160

107. Kokulnathan, T., Joseph Anthuvan, A., Chen, S. M., Chinnuswamy, V., and Kadirvelu, K. (2018) Trace Level Electrochemical Determination of the Neurotransmitter Dopamine in Biological Samples Based on Iron Oxide Nanoparticle Decorated Graphene Sheets. Inorg. Chem. Front. 5 (3), 705-718, DOI: 10.1039/C7QI00716G

108. Reddy, S., Kumara Swamy, B. E., and Jayadevappa, H. (2012) CuO Nanoparticle Sensor for the Electrochemical Determination of Dopamine. Electrochim. Acta 61, 78- 86, DOI: 10.1016/j.electacta.2011.11.091

109. Fayemi, O. E., Adekunle, A. S., Kumara Swamy, B. E., and Ebenso, E. E. (2018) Electrochemical Sensor for the Detection of Dopamine in Real Samples Using Polyaniline/NiO, $\mathrm{ZnO}$, and Fe3O4 Nanocomposites on Glassy Carbon Electrode. J. Electroanal. Chem. 818, 236-249, DOI: 10.1016/j.jelechem.2018.02.027

110. Liu, Q., Zhu, X., Huo, Z., He, X., Liang, Y., and Xu, M. (2012) Electrochemical Detection of Dopamine in the Presence of Ascorbic Acid Using PVP/Graphene Modified

Electrodes. Talanta 97 (10), 557-562, DOI: 10.1016/j.talanta.2012.05.013

111. Wang, Y., Li, Y., Tang, L., Lu, J., and Li, J. (2009) Application of Graphene-Modified Electrode for Selective Detection of 
Dopamine. Electrochem. Commun. 11 (4), 889- 892, DOI: 10.1016/j.elecom.2009.02.013

112. Wu, L., Feng, L., Ren, J., and Qu, X. (2012) Electrochemical Detection of Dopamine Using Porphyrin-Functionalized Graphene. Biosens.

Bioelectron. 34 (1), 57-62, DOI: 10.1016/j.bios.2012.01.007

113. Sun, C. L., Lee, H. H., Yang, J. M., and Wu, C. C. (2011) The Simultaneous Electrochemical Detection of Ascorbic Acid, Dopamine, and Uric Acid Using Graphene/Size-Selected Pt Nanocomposites. Biosens. Bioelectron. 26 (8), 3450-3455, DOI: 10.1016/j.bios.2011.01.023

114. Palanisamy, S., Ku, S., and Chen, S. M. (2013) Dopamine Sensor Based on a Glassy Carbon Electrode Modified with a Reduced Graphene Oxide and Palladium Nanoparticles Composite. Microchim. Acta 180 (1112), 1037-1042, DOI: 10.1007/s00604-013-1028-1

115. Chu, K., Wang, F., Zhao, X.-I., Wang, X.-W., and Tian, Y. (2017) Electrochemical Dopamine Sensor Based on P-Doped Graphene: Highly Active Metal-Free Catalyst and Metal Catalyst Support. Mater. Sci. Eng., C 81, 452- 458, DOI: 10.1016/j.msec.2017.08.053

116. Goyal, R. N., Gupta, V. K., Oyama, M., and Bachheti, N. (2007) Gold Nanoparticles Modified Indium Tin Oxide Electrode for the Simultaneous Determination of Dopamine and Serotonin: Application in Pharmaceutical Formulations and Biological Fluids. Talanta 72 (3), 976-983, DOI: 10.1016/j.talanta.2006.12.029

117. Hannah, S., Al-Hatmi, M., Gray, L., and Corrigan, D. K. (2020) LowCost, Thin-Film, Mass-Manufacturable Carbon Electrodes for Detection of the Neurotransmitter Dopamine. Bioelectrochemistry 133, 107480, DOI: 10.1016/j.bioelechem.2020.107480

118. Gyermek, L. (1996) Pharmacology of Serotonin as Related to Anesthesia. J. Clin. Anesth. 8 (5), 402- 425, DOI: 10.1016/09528180(96)00093-1

119. Wu, K., Fei, J., and Hu, S. (2003) Simultaneous Determination of Dopamine and Serotonin on a Glassy Carbon Electrode Coated with a Film of Carbon Nanotubes. Anal. Biochem. 318 (1), 100-106, DOI: 10.1016/S00032697(03)00174-X

120. Han, H. S., You, J. M., Jeong, H., and Jeon, S. (2013) Synthesis of Graphene Oxide Grafted Poly(Lactic Acid) with Palladium Nanoparticles and Its Application to Serotonin Sensing. Appl. Surf. Sci. 284, 438- 445, DOI: 10.1016/j.apsusc.2013.07.116 
121. Xue, C., Wang, X., Zhu, W., Han, Q., Zhu, C., Hong, J., Zhou, X., and Jiang, H. (2014) Electrochemical Serotonin Sensing Interface Based on Double-Layered Membrane of Reduced Graphene Oxide/Polyaniline Nanocomposites and Molecularly Imprinted Polymers Embedded with Gold Nanoparticles. Sens. Actuators, B 196, 57-63, DOI:

10.1016/j.snb.2014.01.100

122. Selvarajan, S., Suganthi, A., and Rajarajan, M. (2018) A Novel Highly Selective and Sensitive Detection of Serotonin Based on Ag/Polypyrrole/Cu2O Nanocomposite Modified Glassy Carbon Electrode. Ultrason.

Sonoche. 44, 319-330, DOI: 10.1016/j.ultsonch.2018.02.038

123. Tertis, M., Cernat, A., Lacatis, D., Florea, A., Bogdan, D., Suciu, M., Sandulescu, R., and Cristea, C. (2017) Highly Selective Electrochemical Detection of Serotonin on Polypyrrole and Gold Nanoparticles-Based 3D Architecture. Electrochem. Commun. 75, 43- 47, DOI:

10.1016/j.elecom.2016.12.015

124. Dinesh, B., Veeramani, V., Chen, S. M., and Saraswathi, R. (2017) In Situ Electrochemical Synthesis of Reduced Graphene Oxide-Cobalt Oxide Nanocomposite Modified Electrode for Selective Sensing of Depression Biomarker in the Presence of Ascorbic Acid and Dopamine. J. Electroanal. Chem. 786, 169-176, DOI: 10.1016/j.jelechem.2017.01.022

125. Kim, S. K., Kim, D., and Jeon, S. (2012) Electrochemical Determination of Serotonin on Glassy Carbon Electrode Modified with Various Graphene Nanomaterials. Sens. Actuators, B 174, 285- 291, DOI:

10.1016/j.snb.2012.08.034

126. Sadanandhan, N. K., Cheriyathuchenaaramvalli, M., Devaki, S. J., and Ravindranatha Menon, A. R. (2017) PEDOT-Reduced Graphene OxideSilver Hybrid Nanocomposite Modified Transducer for the Detection of Serotonin. J. Electroanal. Chem. 794 (March), 244- 253, DOI: 10.1016/j.jelechem.2017.04.027

127. Fayemi, O. E., Adekunle, A. S., and Ebenso, E.

E. (2017) Electrochemical Determination of Serotonin in Urine Samples Based on Metal Oxide Nanoparticles/MWCNT on Modified Glassy Carbon

Electrode. Sens. Bio-Sensing Res. 13, 17-27, DOI:

0.1016/j.sbsr.2017.01.005

128. Gong, J.-M. and Lin, X.-Q. (2004) Electrochemical Determination of Serotonin and the Competitive Adsorption with Dopamine at 5,5-Ditetradecyl2-(2-Trimethylammonioethyl)-1,3-Dioxane Bromide Lipid Film Modified by Glassy Carbon Electrode. Anal. Sci. 20 (6), 905- 909, DOI: 10.2116/analsci.20.905 
129. Babaei, A. and Babazadeh, M. (2011) A Selective Simultaneous Determination of Levodopa and Serotonin Using a Glassy Carbon Electrode Modified with Multiwalled Carbon Nanotube/Chitosan Composite. Electroanalysis 23 (7), 1726- 1735, DOI: 10.1002/elan.201000755

130. Ran, G., Chen, X., and Xia, Y. (2017) Electrochemical Detection of Serotonin Based on a Poly(Bromocresol Green) Film and Fe3O4 Nanoparticles in a Chitosan Matrix. RSC Adv. 7(4), 1847-1851, DOI: 10.1039/C6RA25639B

131. Satyanarayana, M., Koteshwara Reddy, K., and Vengatajalabathy Gobi, K. (2014) Nanobiocomposite Based Electrochemical Sensor for Sensitive Determination of Serotonin in Presence of Dopamine, Ascorbic Acid and Uric Acid In Vitro. Electroanalysis 26 (11), 2365-2372, DOI:

10.1002/elan.201400243

132. Li, Y. T., Tang, L. N., Ning, Y., Shu, Q., Liang, F. X., Wang, H., and Zhang, G. J. (2016) In Vivo Monitoring of Serotonin by Nanomaterial Functionalized Acupuncture Needle. Sci. Rep. 6 (June), 1-8, DOI:

10.1038/srep28018

133. Hu, W. and Chen, Z. (2017) The Roles of Histamine and Its Receptor Ligands in Central Nervous System Disorders: An Update. Pharmacol. Ther. 175, 116-132, DOI: 10.1016/j.pharmthera.2017.02.039

134. Lin, J.-S., Sakai, K., Vanni-Mercier, G., and Jouvet, M. (1989) A Critical Role of the Posterior Hypothalamus in the Mechanisms of Wakefulness Determined by Microinjection of Muscimol in Freely Moving Cats. Brain Res. 479 (2), 225-240, DOI: 10.1016/0006-8993(89)91623-5

135. Shan, L., Swaab, D. F., and Bao, A. M. (2013) Neuronal Histaminergic System in Aging and Age-Related Neurodegenerative Disorders. Exp. Gerontol. 48 (7), 603-607, DOI: 10.1016/j.exger.2012.08.002

136. Barata-Antunes, S., Cristóvão, A. C., Pires, J., Rocha, S. M., and Bernardino, L. (2017) Dual Role of Histamine on Microglia-Induced Neurodegeneration. Biochim. Biophys. Acta, Mol. Basis

Dis. 1863 (3), 764- 769, DOI: 10.1016/j.bbadis.2016.12.016

137. Luo, L., Yang, J. Y., Xiao, Z. L., Zeng, D. P., Li, Y. J., Shen, Y. D., Sun, Y. M., Lei, H. T., Wang, H., and Xu, Z. L. (2015) A Sensitivity-Enhanced Heterologous Immunochromatographic Assay Based on a Monoclonal Antibody for the Rapid Detection of Histamine in Saury Samples. RSC Adv. 5, 78833- 78840, DOI: 10.1039/C5RA15925C [Crossref], [CAS], Google Scholaropen URL 
138. 138Dong, X.-X., Yang, J.-Y., Luo, L., Zhang, Y.-F., Mao, C., Sun, Y.M., Lei, H.-T., Shen, Y.-D., Beier, R. C., and Xu, Z.-L. (2017) Portable Amperometric Immunosensor for Histamine Detection Using Prussian BlueChitosan-Gold Nanoparticle Nanocomposite Films. Biosens.

Bioelectron. 98, 305- 309, DOI: 10.1016/j.bios.2017.07.014 [Crossref], [PubMed], [CAS], Google Scholaropen URL

139. 139Gajjala, R. K. R. and Palathedath, S. K. (2018) Cu@Pd Core-Shell Nanostructures for Highly Sensitive and Selective Amperometric Analysis of Histamine. Biosens. Bioelectron. 102, 242- 246, DOI:

10.1016/j.bios.2017.11.038 [Crossref], [PubMed], [CAS], Google Scholaropen $\underline{\underline{U R L}}$

140. 140 Geto, A., Tessema, M., and Admassie, S. (2014) Determination of Histamine in Fish Muscle at Multi-Walled Carbon Nanotubes Coated Conducting Polymer Modified Glassy Carbon Electrode. Synth.

Met. 191, 135- 140, DOI: 10.1016/j.synthmet.2014.03.005 [Crossref], [CAS], Google Scholaropen URL

141. 141Kumar, N. and Goyal, R. N. (2018) Silver Nanoparticles Decorated Graphene Nanoribbon Modified Pyrolytic Graphite Sensor for Determination of Histamine. Sens. Actuators, B 268, 383-391, DOI:

10.1016/j.snb.2018.04.136

142. Yang, M., Zhang, J., and Chen, X. (2015) Competitive Electrochemical Immunosensor for the Detection of Histamine Based on Horseradish

Peroxidase Initiated Deposition of Insulating Film. J. Electroanal.

Chem. 736, 88- 92, DOI: 10.1016/j.jelechem.2014.11.002

143. 143Delle, L. E., Huck, C., Backer, M., Muller, F., Grandthyll, S., Jacobs, K., Lilischkis, R., Vu, X. T., Schoning, M. J., Wagner, P., Thoelen, R., Weil, M., and Ingebrandt, S. (2015) Impedimetric Immunosensor for the Detection of Histamine Based on Reduced Graphene Oxide. Phys. Status Solidi A 212 (6), 1327-1334, DOI: 10.1002/pssa.201431863

144. 144Apetrei, M. I. and Apetrei, C. (2016) Amperometric Biosensor Based on Diamine Oxidase/Platinum Nanoparticles/Graphene/Chitosan Modified Screen-Printed Carbon Electrode for Histamine Detection. Sensors 16, 422, DOI: 10.3390/s16040422

145. 145Bao, L., Sun, D., Tachikawa, H., and Davidson, V. L. (2002) Improved Sensitivity of a Histamine Sensor Using an Engineered Methylamine Dehydrogenase. Anal. Chem. 74 (5), 1144-1148, DOI: 10.1021/ac0106086 
146. 146Castro, S. S. L., de Oliveira, M. F., and Stradiotto, N.

R. (2010) Study of the Electrochemical Behavior of Histamine Using a Nafion®-Copper (II) Hexacyanoferrate Film-Modified Electrode. Int. J. Electrochem. Sci. 5 (10), 1447- 1456

147. 147Torre, R., Costa-Rama, E., Lopes, P., Nouws, H. P. A., and Delerue-Matos, C. (2019) Amperometric Enzyme Sensor for the Rapid Determination of Histamine. Anal. Methods 11 (9), 1264-1269, DOI: 10.1039/C8AY02610F

148. 148Ye, W., Xu, Y., Zheng, L., Zhang, Y., Yang, M., and Sun, P. (2016) A Nanoporous Alumina Membrane Based Electrochemical Biosensor for Histamine Determination with Biofunctionalized Magnetic Nanoparticles Concentration and Signal Amplification. Sensors 16, 1767, DOI: 10.3390/s16101767

149. Stojanović, Z. S., Mehmeti, E., Kalcher, K., Guzsvány, V., and Stanković, D. M. (2016) SWCNT-Modified Carbon Paste Electrode as an Electrochemical Sensor for Histamine Determination in Alcoholic Beverages. Food Anal. Methods 9 (10), 2701-2710, DOI: 10.1007/s12161016-0452-3

150. Akhoundian, M., Rüter, A., and Shinde, S. (2017) Ultratrace Detection of Histamine Using a Molecularly-Imprinted Polymer-Based Voltammetric Sensor. Sensors 17, 645, DOI: 10.3390/s17030645

151. Zeng, K., Tachikawa, H., Zhu, Z., and Davidson, V. L. (2000) Amperometric Detection of Histamine with a Methylamine Dehydrogenase Polypyrrole-Based Sensor. Anal. Chem. 72 (10), 2211-2215, DOI: 10.1021/ac9911138

152. Pihel, K., Hsieh, S., Jorgenson, J. W., and Wightman, R.

M. (1995) Electrochemical Detection of Histamine and 5-Hydroxytryptamine at Isolated Mast Cells. Anal. Chem. 67 (24), 4514- 4521, DOI:

10.1021/ac00120a014

153. Niwa, O., Kurita, R., Hayashi, K., Horiuchi, T., Torimitsu, K., Maeyama, K., and Tanizawa, K. (2000) Continuous Measurement of Histamine from Rat Basophilic Leukemia Cells (RBL-2H3) with an on-Line Sensor Using Histamine Oxidase. Sens. Actuators, B 67, 43- 51, DOI: 10.1016/S09254005(00)00339-7

154. Hadi, M. and Mostaanzadeh, H. (2018) Sensitive Detection of Histamine at Metal-Organic Framework (Ni-BTC) Crystals and Multi-Walled Carbon Nanotubes Modified Glassy Carbon Electrode. Russ. J. Electrochem. 54 (12), 1045-1052, DOI: 10.1134/S1023193518120066 
155. Dong, H., Wang, S., Galligan, J. J., and Swain, G. M. (2011) BoronDoped Diamond Nano/Microelectrodes for Biosensing and in Vitro Measurements. Front. Biosci., Scholar Ed. S3, 518- 540, DOI: 10.2741/s169

156. Samaranayake, S., Abdalla, A., Robke, R., Wood, K. M., Zeqja, A., and Hashemi, P. (2015) In Vivo Histamine Voltammetry in the Mouse Premammillary Nucleus. Analyst 140 (11), 3759-3765, DOI: 10.1039/C5AN00313J

157. Puthongkham, P., Lee, S. T., and Venton, B. J. (2019) Mechanism of Histamine Oxidation and Electropolymerization at Carbon Electrodes. Anal. Chem. 91 (13), 8366- 8373, DOI: 10.1021/acs.analchem.9b01178

158. Tsele, T. P., Adekunle, A. S., Fayemi, O. E., and Ebenso, E. E. (2017) Electrochemical Detection of Epinephrine Using Polyaniline Nanocomposite Films Doped with TiO2 and RuO2 Nanoparticles on MultiWalled Carbon Nanotube. Electrochim. Acta 243, 331-348, DOI: 10.1016/j.electacta.2017.05.031

159. Koteshwara Reddy, K., Satyanarayana, M., Yugender Goud, K., Vengatajalabathy Gobi, K., and Kim, H. (2017) Carbon Nanotube Ensembled Hybrid Nanocomposite Electrode for Direct Electrochemical Detection of Epinephrine in Pharmaceutical Tablets and Urine. Mater. Sci. Eng., C 79, 93-99, DOI: 10.1016/j.msec.2017.05.012

160. Ding, M., Zhou, Y., Liang, X., Zou, H., Wang, Z., Wang, M., and Ma, J. (2016) An Electrochemical Sensor Based on Graphene/Poly(Brilliant Cresyl Blue) Nanocomposite for Determination of Epinephrine. J. Electroanal.

Chem. 763, 25-31, DOI: 10.1016/j.jelechem.2015.12.040

161. Thiagarajan, S., Yang, R. F., and Chen, S. M. (2009) Palladium Nanoparticles Modified Electrode for the Selective Detection of Catecholamine Neurotransmitters in Presence of Ascorbic

Acid. Bioelectrochemistry 75, 163-169, DOI:

10.1016/j.bioelechem.2009.03.014

162. Łuczak, T. (2009) Comparison of Electrochemical Oxidation of Epinephrine in the Presence of Interfering Ascorbic and Uric Acids on Gold Electrodes Modified with S-Functionalized Compounds and Gold Nanoparticles. Electrochim. Acta 54 (24), 5863- 5870, DOI: 10.1016/j.electacta.2009.05.047

163. Wierzbicka, E. and Sulka, G. D. (2016) Fabrication of Highly Ordered Nanoporous Thin Au Films and Their Application for Electrochemical Determination of Epinephrine. Sens. Actuators, B 222, 270-279, DOI: 10.1016/j.snb.2015.08.066 
164. Shankar, S. S., Shereema, R. M., and Rakhi, R.

B. (2018) Electrochemical Determination of Adrenaline Using MXene/Graphite Composite Paste Electrodes. ACS Appl. Mater.

Interfaces 10 (50), 43343-43351, DOI: 10.1021/acsami.8b11741

165. Tashkhourian, J., Nami-Ana, S. F., and Shamsipur, M. (2018) Designing a Modified Electrode Based on Graphene Quantum Dot-Chitosan Application to Electrochemical Detection of Epinephrine. J. Mol. Liq. 266, 548- 556, DOI: 10.1016/j.molliq.2018.06.093

166. Thomas, T., Mascarenhas, R. J., Martis, P., Mekhalif, Z., and Swamy, B. E. K. (2013) Multi-Walled Carbon Nanotube Modified Carbon Paste Electrode as an Electrochemical Sensor for the Determination of Epinephrine in the Presence of Ascorbic Acid and Uric Acid. Mater. Sci. Eng., C 33 (6), 3294-3302, DOI: 10.1016/j.msec.2013.04.010

167. Li, J., Wang, X., Duan, H., Wang, Y., and Luo, C. (2016) Ultra-Sensitive Determination of Epinephrine Based on TiO2-Au Nanoclusters Supported on Reduced Graphene Oxide and Carbon Nanotube Hybrid Nanocomposites. Mater. Sci. Eng., C 64, 391-398, DOI: 10.1016/j.msec.2016.04.003

168. Jahanbakhshi, M. (2017) Mesoporous Carbon Foam, Synthesized via Modified Pechini Method, in a New Dispersant of Salep as a Novel Substrate for Electroanalytical Determination of Epinephrine in the Presence of Uric Acid. Mater. Sci. Eng., C 70, 544- 551, DOI: 10.1016/j.msec.2016.09.013

169. Beitollahi, H. and Garkani Nejad, F. (2016) Graphene Oxide/ZnO Nano Composite for Sensitive and Selective Electrochemical Sensing of Levodopa and Tyrosine Using Modified Graphite Screen Printed Electrode. Electroanalysis 28 (9), 2237-2244, DOI: 10.1002/elan.201600143

170. Immanuel, S. and Sivasubramanian, R. (2020) Fabrication of TwoDimensional Chemically Reduced Graphene Oxide Nanosheets for the Electrochemical Determination of Epinephrine. Bull. Mater.

Sci. 43 (1), 79, DOI: 10.1007/s12034-019-2034-7

171. Docherty, J. R. (2008) Pharmacology of Stimulants Prohibited by the World Anti-Doping Agency (WADA). Br. J.

Pharmacol. 154 (3), 606-622, DOI: 10.1038/bjp.2008.124

172. Park, J., Bhimani, R. V., and Bass, C. E. (2018) Review - In Vivo Electrochemical Measurements of Norepinephrine in the Brain: Current Status and Remaining Challenges. J. Electrochem.

Soc. 165 (12), G3051-G3056, DOI: 10.1149/2.0091812jes 
173. Samdani, K. J., Joh, D. W., Rath, M. K., and Lee, K.

T. (2017) Electrochemical Mediatorless Detection of Norepinephrine Based on MoO3 Nanowires. Electrochim. Acta 252, 268-274, DOI:

10.1016/j.electacta.2017.08.187

174. Rosy, Yadav, S. K., Agrawal, B., Oyama, M., and Goyal, R.

N. (2014) Graphene Modified Palladium Sensor for Electrochemical Analysis of Norepinephrine in Pharmaceuticals and Biological Fluids. Electrochim. Acta 125, 622-629, DOI: 10.1016/j.electacta.2014.01.160

175. Nalewajko, E., Wiszowata, A., and Kojło, A. (2007) Determination of Catecholamines by Flow-Injection Analysis and High-Performance Liquid Chromatography with Chemiluminescence Detection. J. Pharm. Biomed. Anal. 43 (5), 1673-1681, DOI: 10.1016/j.jpba.2006.12.021

176. Sun, Y., Tang, Y., Zheng, X., Yao, H., and Xu, Z. (2004) Determination of Catecholamines by Flow Injection Chemiluminescence Method Based on Their Restraining Effects on the Luminol-Potassium Chlorate System. Anal. Lett. 37 (12), 2445-2458, DOI: 10.1081/AL-200029371

177. Allen, S. A., Rednour, S., Shepard, S., and Pond, B. B. (2017) A Simple and Sensitive High-Performance Liquid Chromatography-Electrochemical Detection Assay for the Quantitative Determination of Monoamines and Respective Metabolites in Six Discrete Brain Regions of Mice. Biomed. Chromatogr. 31 (11), e3998 DOI: 10.1002/bmc.3998

178. Zhu, M., Huang, X., Li, J., and Shen, H. (1997) Peroxidase-Based Spectrophotometric Methods for the Determination of Ascorbic Acid, Norepinephrine, Epinephrine, Dopamine and Levodopa. Anal. Chim. Acta 357 (3), 261-267, DOI: 10.1016/S0003-2670(97)00561-8

179. Lin, Z., Wu, X., Lin, X., and Xie, Z. (2007) End-Column Chemiluminescence Detection for Pressurized Capillary Electrochromatographic Analysis of Norepinephrine and Epinephrine. J. Chromatogr. A 1170 (1), 118-121, DOI: 10.1016/j.chroma.2007.09.015

180. Kuhlenbeck, D. L., O'Neill, T. P., Mack, C. E., Hoke, S. H., II, and Wehmeyer, K. R. (2000) Determination of Norepinephrine in Small Volume Plasma Samples by Stable-Isotope Dilution Gas ChromatographyTandem Mass Spectrometry with Negative Ion Chemical Ionization. J. Chromatogr., Biomed. Appl. 738 (2), 319-330, DOI: 10.1016/S03784347(99)00541-1

181. Oka, K., Sekiya, M., Osada, H., Fujita, K., Kato, T., and Nagatsu, T. (1982) Simultaneous Fluorometry of Urinary Dopamine, Norepinephrine, 
and Epinephrine Compared with Liquid Chromatography with Electrochemical Detection. Clin. Chem. 28, 646- 649, DOI: 10.1093/clinchem/28.4.646

182. Wang, Z., Wang, K., Zhao, L., Chai, S., Zhang, J., Zhang, X., and Zou, Q. (2017) A Novel Sensor Made of Antimony Doped Tin Oxide-Silica Composite Sol on a Glassy Carbon Electrode Modified by Single-Walled Carbon Nanotubes for Detection of Norepinephrine. Mater. Sci. Eng., C 80, 180-186, DOI: 10.1016/j.msec.2017.03.227

183. Ma, X., Chen, M., Li, X., Purushothaman, A., and Li, F. (2012) Electrochemical Detection of Norepinephrine in the Presence of Epinephrine, Uric Acid and Ascorbic Acid Using a Graphene-Modified Electrode. Int. J. Electrochem. Sci. 7 (2), 991-1000, DOI: 10.1039/c2ay25040c

184. Vilian, A. T. E., Chen, S.-M., Hung, Y.-T., Ali, M. A., and Al-Hemaid, F. M. A. (2014) Electrochemical Oxidation and Determination of Norepinephrine in the Presence of Acetaminophen Using MnO2 Nanoparticle Decorated Reduced Graphene Oxide Sheets. Anal. Methods 6 (16), 6504-6513, DOI: 10.1039/C4AY00878B

185. Anandaraj, S., Chen, T. W., Chen, S. M., Elaiyappillai, E., Loganathan, S., Ibrahimsa, S. L., Johnson, P. M., Selvin, S. P., Weng, W. H., and Leung, W. H. (2017) Electrochemical Detection of Norepinephrine Using Sponge-like Co3O4Modified Screen Printed Carbon Electrode. Int. J. Electrochem. Sci. 12 (11), 10524- 10533, DOI: 10.20964/2017.11.85

186. Lee, E. J., Choi, J. H., Um, S. H., and Oh, B. K. (2017) Electrochemical Sensor for Selective Detection of Norepinephrine Using Graphene SheetsGold Nanoparticle Complex Modified Electrode. Korean J. Chem.

Eng. 34 (4), 1129-1132, DOI: 10.1007/s11814-016-0363-4

187. Mazloum-Ardakani, M., Beitollahi, H., and Kazem, M. (2010) Sensors and Actuators B: Chemical New Strategy for Simultaneous and Selective Voltammetric Determination of Norepinephrine, Acetaminophen and Folic Acid Using ZrO 2 Nanoparticles-Modified Carbon Paste Electrode. Sensors Actuators B. Chem. 151 (1), 243-249, DOI: 10.1016/j.snb.2010.09.011

188. Mazloum-Ardakani, M., Beitollahi, H., Sheikh-Mohseni, M. A., Naeimi, H., and Taghavinia, N. (2010) Novel Nanostructure Electrochemical Sensor for Electrocatalytic Determination of Norepinephrine in the Presence of High Concentrations of Acetaminophene and Folic Acid. Appl. Catal., A 378 (2), 195- 201, DOI: 10.1016/j.apcata.2010.02.019

189. Bian, C., Zeng, Q., Xiong, H., Zhang, X., and Wang, S. (2010) Electrochemistry of Norepinephrine on Carbon-Coated Nickel 
Magnetic Nanoparticles Modified Electrode and Analytical Applications. Bioelectrochemistry 79 (1), 1-5, DOI:

10.1016/j.bioelechem.2009.09.011

190. Lu, L. P., Wang, S. Q., and Lin, X. Q. (2004) Fabrication of Layer-byLayer Deposited Multilayer Films Containing DNA and Gold Nanoparticle for Norepinephrine Biosensor. Anal. Chim. Acta 519, 161-166, DOI:

10.1016/j.aca.2004.05.062

191. Samdani, K. J., Samdani, J. S., Kim, N. H., and Lee, J.

H. (2016) FeMoO4 Based, Enzyme-Free Electrochemical Biosensor for Ultrasensitive Detection of Norepinephrine. Biosens.

Bioelectron. 81, 445- 453, DOI: 10.1016/j.bios.2016.03.029

192. Nasirizadeh, N. and Zare, H. R. (2009) Differential Pulse Voltammetric Simultaneous Determination of Noradrenalin and Acetaminophen Using a Hematoxylin Biosensor. Talanta 80 (2), 656- 663, DOI:

10.1016/j.talanta.2009.07.044

193. Chen, W., Lin, X., Luo, H., and Huang, L. (2005) Electrocatalytic Oxidation and Determination of Norepinephrine at Poly(Cresol Red) Modified Glassy Carbon Electrode. Electroanalysis 17, 941- 945, DOI: 10.1002/elan.200403199

194. de Queiroz, D. F., de Lima Dadamos, T. R. L., Machado, S. A. S., and Martines, M. A. U. (2018) Electrochemical Determination of Norepinephrine by Means of Modified Glassy Carbon Electrodes with Carbon Nanotubes and Magnetic Nanoparticles of Cobalt

Ferrite. Sensors 18 (4), 1223, DOI: 10.3390/s18041223

195. D., Shi, Z., Liu, Z., Low, S. S., Zhu, J., Zhang, T., Chen, Z., Yu, X., Lu, Y., Lu, D., and Liu, Q. (2020) Smartphone-Based Square Wave Voltammetry System with Screen-Printed Graphene Electrodes for Norepinephrine Detection. Smart Mater. Med. 1, 1- 9, DOI: 10.1016/j.smaim.2020.02.001

196. Chen, J., Huang, H., Zeng, Y., Tang, H., and Li, L. (2015) A Novel Composite of Molecularly Imprinted Polymer-Coated PdNPs for Electrochemical Sensing Norepinephrine. Biosens.

Bioelectron. 65, 366- 374, DOI: 10.1016/j.bios.2014.10.011

197. Kleppner, S. R. and Tobin, A. J. (2001) GABA Signalling: Therapeutic Targets for Epilepsy, Parkinson's Disease and Huntington's Disease. Emerging Ther. Targets 5 (2), 219-239, DOI: 10.1517/14728222.5.2.219 
198. Prasad, B. B., Prasad, A., and Tiwari, M. P. (2013) Highly Selective and Sensitive Analysis of $y$-Aminobutyric Acid Using a New Molecularly Imprinted Polymer Modified at the Surface of Abrasively Immobilized Multi-Walled Carbon Nanotubes on Pencil Graphite Electrode. Electrochim.

Acta 102, 400- 408, DOI: 10.1016/j.electacta.2013.04.043

199. Iwai, N. T., Kramaric, M., Crabbe, D., Wei, Y., Chen, R., and Shen, M. (2018) GABA Detection with Nano-ITIES Pipet Electrode: A New Mechanism, Water/DCE-Octanoic Acid Interface. Anal.

Chem. 90 (5), 3067-3072, DOI: 10.1021/acs.analchem.7b03099

200. Barman, K. and Jasimuddin, S. (2016) Simultaneous Electrochemical Detection of Dopamine and Epinephrine in the Presence of Ascorbic Acid and Uric Acid Using a AgNPs-Penicillamine-Au Electrode. RSC Adv. 6 (102), 99983- 99988, DOI: 10.1039/C6RA19813A

201. Selvaraju, T. and Ramaraj, R. (2003) Simultaneous Determination of Ascorbic Acid, Dopamine and Serotonin at Poly(Phenosafranine) Modified Electrode. Electrochem. Commun. 5 (8), 667-672, DOI: 10.1016/S13882481(03)00151-6

202. Wang, Y., Wang, S., Tao, L., Min, Q., Xiang, J., Wang, Q., Xie, J., Yue, Y., Wu, S., Li, X., and Ding, H. (2015) A Disposable Electrochemical Sensor for Simultaneous Determination of Norepinephrine and Serotonin in Rat Cerebrospinal Fluid Based on MWNTs-ZnO/Chitosan Composites Modified Screen-Printed Electrode. Biosens. Bioelectron. 65, 31- 38, DOI: 10.1016/j.bios.2014.09.099

203. da Silva, L. V., Lopes, C. B., da Silva, W. C., de Paiva, Y. G., Silva de dos, F. A. S., Lima, P. R., Kubota, L. T., and Goulart, M. O.

F. (2017) Electropolymerization of Ferulic Acid on Multi-Walled Carbon Nanotubes Modified Glassy Carbon Electrode as a Versatile Platform for $\mathrm{NADH}$, Dopamine and Epinephrine Separate Detection. Microchem. J. 133, 460-467, DOI: 10.1016/j.microc.2017.04.014

204. Wang, Z. H., Liang, Q. L., Wang, Y. M., and Luo, G. A. (2003) Carbon Nanotube-Intercalated Graphite Electrodes for Simultaneous Determination of Dopamine and Serotonin in the Presence of Ascorbic Acid. J. Electroanal. Chem. 540, 129-134, DOI: 10.1016/S0022-0728(02)01300-1

205. Abbaspour, A. and Noori, A. (2011) A Cyclodextrin Host-Guest Recognition Approach to an Electrochemical Sensor for Simultaneous Quantification of Serotonin and Dopamine. Biosens.

Bioelectron. 26 (12), 4674- 4680, DOI: 10.1016/j.bios.2011.04.061 
206. Zhou, H., Yu, R., Wang, C., Ran, G., Song, Q., and Masson, J. F. (2020) Simple Multistep Assembly of Hybrid Carbon Material Based Microelectrode for Highly Sensitive Detection of Neurotransmitters. J. Electroanal. Chem. 863, 114082, DOI: 10.1016/j.jelechem.2020.114082

207. Li, J. and Lin, X. (2007) Simultaneous Determination of Dopamine and Serotonin on Gold Nanocluster/Overoxidized-Polypyrrole Composite Modified Glassy Carbon Electrode. Sens. Actuators, B 124 (2), 486- 493, DOI: 10.1016/j.snb.2007.01.021

208. Han, H. S., Lee, H. K., You, J. M., Jeong, H., and Jeon, S. (2014) Electrochemical Biosensor for Simultaneous Determination of Dopamine and Serotonin Based on Electrochemically Reduced GOPorphyrin. Sens. Actuators, B 190, 886- 895, DOI: 10.1016/j.snb.2013.09.022

209. Goyal, R. N. and Bishnoi, S. (2011) Simultaneous Determination of Epinephrine and Norepinephrine in Human Blood Plasma and Urine Samples Using Nanotubes Modified Edge Plane Pyrolytic Graphite Electrode. Talanta 84 (1), 78-83, DOI: 10.1016/j.talanta.2010.12.034

210. Mphuthi, N. G., Adekunle, A. S., and Ebenso, E.

E. (2016) Electrocatalytic Oxidation of Epinephrine and Norepinephrine at Metal Oxide Doped Phthalocyanine/MWCNT Composite Sensor. Sci.

Rep. 6 (June), 1-20, DOI: 10.1038/srep26938

211. Mazloum-Ardakani, M. and Khoshroo, A. (2014) High Sensitive Sensor Based on Functionalized Carbon Nanotube/lonic Liquid Nanocomposite for Simultaneous Determination of Norepinephrine and Serotonin. J. Electroanal. Chem. 717-718, 17-23, DOI: 10.1016/j.jelechem.2013.12.034

\section{2. $\underline{\mathbf{2 1 2}}$}

Thiagarajan, S., Yang, R. F., and Chen, S. M. (2009) Palladium Nanoparticles Modified Electrode for the Selective Detection of Catecholamine Neurotransmitters in Presence of Ascorbic Acid. Bioelectrochemistry 75 (2), 163-169, DOI:

10.1016/j.bioelechem.2009.03.014

\section{3. $\underline{\mathbf{2 1 3}}$}


Sarada, B. V., Rao, T. N., Tryk, D. A., and Fujishima,

A. (2000) Electrochemical Oxidation of Histamine and Serotonin at Highly Boron- Doped Diamond Electrodes. Anal. Chem. 72 (7), 1632- 1638, DOI: $10.1021 /$ ac9908748

214. Balasubramanian, P., Balamurugan, T. S. T., Chen, S. M., Chen, T. W., and Sathesh, T. (2019) Rational Design of Cu@Cu2O Nanospheres Anchored B, N Co-Doped Mesoporous Carbon: A Sustainable Electrocatalyst to Assay Eminent Neurotransmitters Acetylcholine and Dopamine. ACS Sustainable Chem. Eng. 7 (6), 5669- 5680, DOI: 10.1021/acssuschemeng.8b04473

215. Atta, N. F., Galal, A., El-Ads, E. H., and Galal, A. E. (2020) Efficient Electrochemical Sensor Based on Gold Nanoclusters/Carbon Ionic Liquid Crystal for Sensitive Determination of Neurotransmitters and Anti-Parkinson Drugs. Adv. Pharm. Bull. 10 (1), 46- 55, DOI: 10.15171/apb.2020.006

216. Gupta, P. and Goyal, R. N. (2014) Polymelamine Modified Edge Plane Pyrolytic Graphite Sensor for the Electrochemical Assay of

Serotonin. Talanta 120,17-22, DOI: 10.1016/j.talanta.2013.11.075

\section{7. $\underline{\mathbf{2 1 7}}$}

Wierzbicka, E. and Sulka, G. D. (2016) Nanoporous Spongelike Au-Ag Films for Electrochemical Epinephrine Sensing. J. Electroanal.

Chem. 762, 43- 50, DOI: 10.1016/j.jelechem.2015.12.013

\section{8. $\underline{\mathbf{2 1 8}}$}

Baniasadi, M., Jahani, S., Maaref, H., and Alizadeh, R. (2017) Voltammetric Determination of Epinephrine Based on ZnO Nanoparticles Assisted Graphene Oxide Nanosheets. Anal. Bioanal. Electrochem. 9, 718- 728

\section{9. $\underline{219}$}

Teradale, A. B., Lamani, S. D., Ganesh, P. S., Kumara Swamy, B. E., and Das, S. N. (2017) Niacin Film Coated Carbon Paste Electrode Sensor for the Determination of Epinephrine in Presence of Uric Acid: A Cyclic Voltammetric Study. Anal. Chem. Lett. 7, 748- 764, DOI: 10.1080/22297928.2017.1396917 
Manuscript version: 20 November 2020

ACS Publications, ACS Chem. Neurosci. https://doi.org/10.1021/acschemneuro.0c00355 